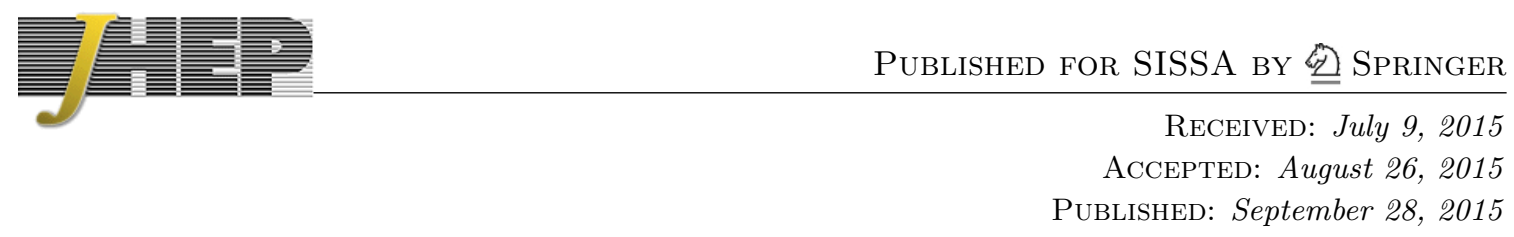

\title{
Parton distributions with threshold resummation
}

\author{
Marco Bonvini, ${ }^{a}$ Simone Marzani, ${ }^{b}$ Juan Rojo, ${ }^{a}$ Luca Rottoli, ${ }^{a}$ Maria Ubiali, ${ }^{c}$ \\ Richard D. Ball, ${ }^{d, e}$ Valerio Bertone, ${ }^{e}$ Stefano Carrazza $^{f}$ and Nathan P. Hartland ${ }^{a}$ \\ ${ }^{a}$ Rudolf Peierls Centre for Theoretical Physics, University of Oxford, \\ 1 Keble Road, OX1 3NP, Oxford, U.K. \\ ${ }^{b}$ Center for Theoretical Physics, Massachusetts Institute of Technology, \\ 77 Massachusetts Ave, Cambridge, MA 02139, U.S.A. \\ ${ }^{c}$ Cavendish Laboratory, HEP group, University of Cambridge, \\ J.J. Thomson Avenue, CB3 OHE, Cambridge, U.K. \\ ${ }^{d}$ The Higgs Centre for Theoretical Physics, University of Edinburgh, \\ JCMB, KB, Mayfield Rd, Edinburgh EH9 3JZ, Scotland, U.K. \\ ${ }^{e} \mathrm{PH}$ Department, TH Unit, CERN, \\ CH-1211 Geneva 23, Switzerland \\ ${ }^{f}$ Dipartimento di Fisica, Università di Milano and INFN, Sezione di Milano, \\ Via Celoria 16, I-20133 Milano, Italy \\ E-mail: marco.bonvini@physics.ox.ac.uk, smarzani@buffalo.edu, \\ juan.rojo@physics.ox.ac.uk, luca.rottoli@physics.ox.ac.uk, \\ ubiali@hep.phy.cam.ac.uk, rdb@ph.ed.ac.uk, valerio.bertone@cern.ch, \\ stefano.carrazza@mi.infn.it, nathan.hartland@physics.ox.ac.uk
}

ABSTRACT: We construct a set of parton distribution functions (PDFs) in which fixed-order NLO and NNLO calculations are supplemented with soft-gluon (threshold) resummation up to NLL and NNLL accuracy respectively, suitable for use in conjunction with any QCD calculation in which threshold resummation is included at the level of partonic cross sections. These resummed PDF sets, based on the NNPDF3.0 analysis, are extracted from deepinelastic scattering, Drell-Yan, and top quark pair production data, for which resummed calculations can be consistently used. We find that, close to threshold, the inclusion of resummed PDFs can partially compensate the enhancement in resummed matrix elements, leading to resummed hadronic cross-sections closer to the fixed-order calculations. On the other hand, far from threshold, resummed PDFs reduce to their fixed-order counterparts. Our results demonstrate the need for a consistent use of resummed PDFs in resummed calculations.

KEYWORDS: Resummation, QCD, Deep Inelastic Scattering

ARXIV EPRINT: 1507.01006 


\section{Contents}

1 Introduction 1

2 Threshold resummation $\quad 4$

2.1 Theoretical framework 4

$\begin{array}{lll}2.2 & \text { Numerical implementation } & 7\end{array}$

3 Settings of the resummed PDF fit $\quad 9$

$\begin{array}{lll}3.1 & \text { Experimental data } & 10\end{array}$

$\begin{array}{ll}3.2 & \text { Calculation of resummed } K \text {-factors } \\ \end{array}$

4 Parton distributions with threshold resummation $\quad 16$

$\begin{array}{lll}4.1 & \text { Baseline fixed-order fits } & 16\end{array}$

$\begin{array}{lll}4.2 & \text { DIS-only resummed PDFs } & 18\end{array}$

$\begin{array}{lll}4.3 & \text { DIS+DY+top resummed PDFs } & 20\end{array}$

$\begin{array}{lll}4.4 & \text { Partonic luminosities } & 22\end{array}$

5 Resummed PDFs: implications for LHC phenomenology 25

5.1 SM and BSM Higgs production in gluon fusion 27

5.2 High-Mass Drell-Yan dilepton mass distributions 28

$\begin{array}{lll}5.3 & \text { Supersymmetric particle production } & 29\end{array}$

$\begin{array}{lll}6 & \text { Summary } & 31\end{array}$

\section{Introduction}

The accurate determination of the parton distribution functions (PDFs) of the proton is an essential ingredient of the LHC physics program [1-5]. In order to reduce theoretical uncertainties, it is crucial to incorporate in global PDF fits higher-order perturbative QCD corrections, both to the hard partonic cross sections and to the parton evolution. While recent progress in fixed-order NLO (see e.g. [6] for a recent review), NNLO (e.g. [7-23]) and even $\mathrm{N}^{3} \mathrm{LO}[24]$ calculations for different processes in hadron-hadron collisions has been impressive, it is also well-known that fixed-order perturbative calculations display classes of logarithmic contributions that become large in some kinematic regions, thus spoiling the perturbative expansion in the strong coupling constant $\alpha_{s}$. The importance of these contributions varies significantly with both the type and the kinematic regime of the processes which enter PDF fits. Therefore, their omission can lead to a significant distortion of the PDFs, thereby reducing their theoretical accuracy (see ref. [25] for a detailed discussion). In order to avoid this problem, it is necessary to supplement fixedorder calculations with all-order resummations of these large logarithms. 
Logarithmic enhancements of higher-order perturbative contributions originate from a number of different kinematic regions and require, in general, different resummation techniques (see e.g. ref. [26] for a recent review). For instance, enhancements may take place when the centre-of-mass energy of the partonic collision is much higher than the hard scale of the process: this corresponds to the small- $x$ region of the PDFs, and the resummation of such terms is known as high-energy or small- $x$ resummation, see e.g. [27-30]. Small- $x$ resummation is certainly relevant for PDF determination and might be needed to describe the most recent HERA data, where some tensions with fixed-order DGLAP have been reported [31-33]. A study of small- $x$ resummation in PDF fits will be presented elsewhere. In this paper we concentrate instead on another type of logarithmic enhancement of higher order perturbative contributions which appear close to threshold for the production of the final states: this is the large- $x$ kinematic region, and the resummation of logarithms from this region is known as large- $x$, soft gluon, or threshold resummation.

All-order threshold resummations exist for many of the processes which play a central role in the exploration of the electroweak scale being pursued at the LHC. For instance, the current Higgs Cross section Working Group recommendation for the gluon-fusion cross section includes threshold resummation [34, 35], and resummed Higgs cross sections in this channel are available up to $\mathrm{N}^{3} \mathrm{LL}$ [36-38]. Additional resummed calculations for Higgs physics exist, for example for gluon-induced Higgs Strahlung [39] and for Higgs-pair production [40]. The basis for threshold resummation in QCD were laid in refs. [41-43], in which explicit resummed expressions to NLL accuracy were given for processes relevant for global fits of parton distributions, such as deep-inelastic structure functions and DrellYan total cross section. Subsequently, higher-order resummed calculations have been made available for deep-inelastic scattering structure functions [44-48], invariant mass distributions [38, 47-51] and rapidity distributions [52-58] in Drell-Yan production, and top quark pair production, both inclusive [59,60] and differentially [61-63]. As far as processes relevant for New Physics searches are concerned, resummed calculations exist for squark and gluino production [64-67], stop quark pair production [68, 69], slepton and gaugino pair production [70-73] among many others.

Moreover, it is well known [74, 75] that in the commonly used $\overline{\mathrm{MS}}$ scheme, threshold resummation affects only partonic coefficient functions, while the singular part of the DGLAP splitting function is given, to any order in perturbation theory, by the cusp contribution: $P\left(x, \alpha_{s}\right) \sim \Gamma_{\text {cusp }}\left(\alpha_{s}\right) /(1-x)$, as $x \rightarrow 1$. Therefore, to perform a resummed PDF fit it is only necessary to modify the partonic cross sections, while the NLO [76-79] and NNLO $[80,81]$ DGLAP evolution kernels remain unchanged.

Despite the wide range of resummed calculations available, a complete global PDF fit including the effects of threshold resummation has never been produced (although some preliminary results were presented in ref. [82]). A first study, restricted to non-singlet DIS structure functions, was performed in ref. [83], finding that at NLO resummation could suppress the large- $x$ valence quark PDFs by as much as ten percent. The impact of threshold resummation in direct photon production and its implications on the large- $x$ gluon was studied in ref. [84]. More recently, threshold resummation has been studied in the context of the CJ fits [85], with emphasis on the description of the large- $x$ JLAB data. 
Given the impressive theoretical developments in the resummation of hard-scattering cross sections, it is clear that a state-of-the-art resummed global PDF fit is most timely. This is what we plan to achieve in this paper.

To this end we will produce for the first time NLO+NLL and NNLO+NNLL thresholdresummed fits based on the NNPDF methodology [86-93]. Since NNPDF fitting is free from theoretical bias, due to the very flexible PDF parametrisation, it is sufficiently precise to be able to detect even small changes in PDFs due to threshold resummation of the various processes that go into the global fit.

A major obstacle to producing a truly global resummed fit is that for a number of important processes, in particular inclusive jet production and $W$ production at the leptonic level, threshold resummation is not readily available. For inclusive jets, resummed calculations have been used to determine approximate expressions [94-96] for the yet unknown NNLO contributions, but codes that provide all-order results are not publicly available. For $W$ production, resummation is available only at the level of reconstructed $W$ but not for the measured lepton-level distributions.

For this reason, in this work we have begun by producing variants of the NNPDF3.0 global NLO and NNLO fits [97] based only on those processes which can be consistently resummed: fixed-target and collider neutral and charged current deep-inelastic structure functions, fixed-target and collider neutral current Drell-Yan production, and inclusive topquark pair production. These DIS+DY+top fits then provide a suitable baseline to compare with the NLL and NNLL resummed fits. One important drawback is that the resulting fits will be affected by larger PDF uncertainties as compared to the global NNPDF3.0 set, due to the missing experiments, affecting in particular gluon-initiated processes. In this respect, it will be important to produce updated resummed fits as soon as the missing resummed calculations become available.

An important goal of this paper is to quantify the inaccuracies that affect current resummed calculations due to the inconsistent use of a fixed-order PDF with resummed partonic cross sections. As we will show, for final states with large invariant mass, close to the hadronic threshold, the main effect of the resummed PDFs is to bring the resummed hadronic calculation closer to the fixed-order result, thereby canceling partially the effect of the resummation in the matrix elements. On the other hand, for final states far below threshold, such as inclusive Higgs production at the LHC, the effect of the resummation on the PDFs can be small compared with the resummation in the matrix elements. We also find that, unsurprisingly, resummed and unresummed PDFs are much closer at NNLO than at NLO. Our results emphasise the need for a consistent use of resummed PDFs in resummed calculations: the use of fixed-order PDFs with resummed matrix elements can lead to misleading results, particularly at NLO.

The outline of this paper is as follows. In section 2 we review some basic concepts and results in threshold resummation, as well as their implementation. In section 3, we discuss the settings of the global PDF fit used here to include threshold resummation effects, which is a variant of the recent NNPDF3.0 global fit. The results of the resummed fits are then discussed in section 4, where we compare resummed with fixed-order PDFs at $\mathrm{NLO}(+\mathrm{NLL})$ and $\mathrm{NNLO}(+\mathrm{NNLL})$. Then in section 5 we discuss the implications of the 
resummed PDFs for LHC phenomenology, with emphasis on the mismatch that can arise if fixed-order PDFs are used in resummed calculations. Finally we summarise in section 6 and discuss the delivery of the resummed PDFs produced in this work.

\section{Threshold resummation}

In this section we review the theoretical formalism of threshold resummation, and then we discuss its practical implementation in order to be able to use it in the resummed NNPDF fits. We work in the traditional framework of perturbative QCD (see e.g. [98]); alternative results can be obtained using the methods of Soft-Collinear Effective Theory (see ref. [99] for a recent review and refs. [100-103] for more detailed comparisons between the two formalisms).

\subsection{Theoretical framework}

We start by considering a hadron-level cross section

$$
\sigma\left(x, Q^{2}\right)=x \sum_{a, b} \int_{x}^{1} \frac{d z}{z} \mathcal{L}_{a b}\left(\frac{x}{z}, \mu_{\mathrm{F}}^{2}\right) \frac{1}{z} \hat{\sigma}_{a b}\left(z, Q^{2}, \alpha_{s}\left(\mu_{\mathrm{R}}^{2}\right), \frac{Q^{2}}{\mu_{\mathrm{F}}^{2}}, \frac{Q^{2}}{\mu_{\mathrm{R}}^{2}}\right),
$$

where $a, b$ run over parton flavors, $Q^{2}$ is the hard scale of the process, $x$ is a dimensionless variable and $x \rightarrow 1$ defines the threshold limit. For the resummed fit we are going to consider three processes: deep-inelastic scattering (DIS) of a lepton off a hadron, the DrellYan process (DY) and top-anti-top production $(t \bar{t})$. In DIS, $Q^{2}$ is the off-shellness of the exchanged boson $Q^{2}=-q^{2}$ and $x=\frac{Q^{2}}{2 p \cdot q}$, where $p$ is the hadron momentum. In DY, $Q$ is the invariant mass of the lepton pair and $x=\frac{Q^{2}}{s}$, being $\sqrt{s}$ the collider centre-of-mass energy. Finally, for $t \bar{t}, Q^{2}=4 m_{t}^{2}$ and $x=\frac{Q^{2}}{s}$. In eq. (2.1), $\mathcal{L}_{a b}\left(z, \mu^{2}\right)$ is a parton luminosity, defined as

$$
\mathcal{L}_{a b}\left(z, \mu^{2}\right)=\int_{z}^{1} \frac{d w}{w} f_{a}\left(\frac{z}{w}, \mu^{2}\right) f_{b}\left(w, \mu^{2}\right),
$$

in the hadron-hadron collision case, while in the case of DIS it is just a single PDF. In the following we are going to set $\mu_{\mathrm{R}}=\mu_{\mathrm{F}}=Q$.

In order to diagonalise the convolution integral, we take Mellin moments of eq. (2.1):

$$
\sigma\left(N, Q^{2}\right)=\int_{0}^{1} d x x^{N-2} \sigma\left(x, Q^{2}\right)=\sum_{a, b} \mathcal{L}_{a b}\left(N, Q^{2}\right) \hat{\sigma}_{a b}\left(N, Q^{2}, \alpha_{s}\right),
$$

where $\alpha_{s}=\alpha_{s}\left(Q^{2}\right)$ and

$$
\begin{aligned}
\mathcal{L}_{a b}\left(N, Q^{2}\right) & =\int_{0}^{1} d z z^{N-1} \mathcal{L}_{a b}\left(z, Q^{2}\right), \\
\hat{\sigma}_{a b}\left(N, Q^{2}, \alpha_{s}\right) & =\int_{0}^{1} d z z^{N-2} \hat{\sigma}_{a b}\left(z, Q^{2}, \alpha_{s}\right) .
\end{aligned}
$$

In Mellin space the threshold limit corresponds to $N \rightarrow \infty$ and the aim of threshold resummation is to obtain a more reliable estimate of the hadron-level cross section by resumming 
to all orders in the strong coupling $\alpha_{s}$ the logarithmically enhanced contributions to the partonic cross section $\hat{\sigma}_{a b}$ at large $N$. The resummed partonic cross section can be written as the product of a Born contribution and an all-order coefficient function:

$$
\hat{\sigma}_{a b}^{(\mathrm{res})}\left(N, Q^{2}, \alpha_{s}\right)=\sigma_{a b}^{(\mathrm{born})}\left(N, Q^{2}, \alpha_{s}\right) C_{a b}^{(\mathrm{res})}\left(N, \alpha_{s}\right),
$$

where

$$
\begin{aligned}
C_{a b}^{(\mathrm{res})}\left(N, \alpha_{s}\right) & =\sum_{\mathbf{I}} \bar{g}_{0 a b}^{(\mathbf{I})}\left(\alpha_{s}\right) \exp \overline{\mathcal{S}}^{(\mathbf{I})}\left(N, \alpha_{s}\right), \\
\overline{\mathcal{S}}^{(\mathbf{I})}\left(N, \alpha_{s}\right) & =\ln \Delta_{a}+\ln \Delta_{b}+\ln J_{c}+\ln J_{d}+\ln \Delta_{a b \rightarrow c d}^{(\mathbf{I})} .
\end{aligned}
$$

The notation $a b \rightarrow c d$ has been chosen to accommodate all the processes that enter our fit. For $t \bar{t}$ production, we have to consider the resummation of two Born-level processes, namely $q \bar{q} \rightarrow t \bar{t}$ and $g g \rightarrow t \bar{t}$. For DIS instead we have $V^{*} q \rightarrow q$ and for DY $q \bar{q} \rightarrow V^{*}$. Moreover, while in DIS and DY we have one color structure, in the $t \bar{t}$ case we have two contributions, i.e. $\mathbf{I}=$ singlet, octet.

Let us now examine the different contributions to the resummed exponent. If $i$ is a color-singlet, then $\Delta_{i}=J_{i}=1$. For each initial-state QCD parton, we have an initial-state jet function

$$
\ln \Delta_{i}=\int_{0}^{1} d z \frac{z^{N-1}-1}{1-z} \int_{\mu_{\mathrm{F}}^{2}}^{(1-z)^{2} Q^{2}} \frac{d q^{2}}{q^{2}} A_{i}\left(\alpha_{s}\left(q^{2}\right)\right), \quad i=a, b .
$$

For each massless final-state QCD parton we have a final-state jet function

$$
\ln J_{i}=\int_{0}^{1} d z \frac{z^{N-1}-1}{1-z}\left[\int_{(1-z)^{2} Q^{2}}^{(1-z) Q^{2}} \frac{d q^{2}}{q^{2}} A_{i}\left(\alpha_{s}\left(q^{2}\right)\right)+\frac{1}{2} B_{i}\left(\alpha_{s}\left(Q^{2}(1-z)\right)\right)\right], \quad i=c, d,
$$

while there is no jet-function for $t$ or $\bar{t}$. Finally we also have a large-angle soft contribution, which depends in principle on both the process and the color flow:

$$
\ln \Delta_{a b \rightarrow c d}^{(\mathbf{I})}=\int_{0}^{1} d z \frac{z^{N-1}-1}{1-z} D_{a b \rightarrow c d}^{(\mathbf{I})}\left(\alpha_{s}\left(Q^{2}(1-z)^{2}\right)\right) .
$$

The functions $A_{i}\left(\alpha_{s}\right), B_{i}\left(\alpha_{s}\right), D_{i}^{(\mathbf{I})}\left(\alpha_{s}\right)$, and obviously $\bar{g}_{0}^{(\mathbf{I})}\left(\alpha_{s}\right)$, are free of large logarithms and can be computed in fixed-order perturbation theory. The accuracy of their determination fixes the logarithmic accuracy of the resummation. In particular, (N)NLL requires $A_{i}$ to second (third) order in the strong coupling $\alpha_{s}$, and $B_{i}, D_{i}^{(\mathbf{I})}$, and $\bar{g}_{0 a b}$ to first (second) order. ${ }^{1}$ Threshold resummation is actually known to $\mathrm{N}^{3}$ LL for DIS [45] and DY [38, 49-51], and to NNLL for $t \bar{t}$ production [59, 104].

We have left out of our discussion inclusive jet production. ${ }^{2}$ The general framework to perform this resummation has been worked out long ago [105]. However, different treatments of the jet kinematics at threshold can lead to substantially different results, see

\footnotetext{
${ }^{1}$ This accuracy is sometimes referred to as (N)NLL'. For a precise definition of all possible accuracies and their nomenclature in threshold resummation, see table 1 of ref. [36].

${ }^{2}$ We acknowledge discussions with Mrinal Dasgupta and Werner Vogelsang on this topic.
} 
e.g. ref. [94] and [106]. Moreover, depending on the way the threshold limit is defined, NLL resummation can be affected by non-global logarithms [107] and the result may acquire a non-trivial dependence on the jet algorithm [108, 109]. In addition, as previously mentioned, computer programs that implement threshold resummation for jet production are not, to the best of our knowledge, publicly available. On the other hand, recent progress [110-112] has shown that NNLL accuracy is perhaps achievable in the near future. We leave a detailed phenomenological analysis of jet production and its inclusion in a PDF fit to future work.

The Mellin integrals in the resummed expression eq. (2.6) are often evaluated in the $N \rightarrow \infty$ limit, thereby keeping only those contributions that do not vanish at large $N$ and behave as powers of $\ln N$. In this approximation, which we refer to as $N$-soft in the following, the resummed coefficient function becomes

$$
\begin{aligned}
C^{(N \text {-soft })}\left(N, \alpha_{s}\right) & =g_{0}\left(\alpha_{s}\right) \exp \mathcal{S}\left(\ln N, \alpha_{s}\right), \\
\mathcal{S}\left(\ln N, \alpha_{s}\right) & =\left[\frac{1}{\alpha_{s}} g_{1}\left(\alpha_{s} \ln N\right)+g_{2}\left(\alpha_{s} \ln N\right)+\alpha_{s} g_{3}\left(\alpha_{s} \ln N\right)+\ldots\right],
\end{aligned}
$$

where, in order to simplify our notation, we henceforth drop all the flavor and color-flow indices and it is understood that all the modifications we discuss are applied to each partonic subprocess and each color-flow. The functions $g_{i}\left(\alpha_{s} \ln N\right)$ with $i \geq 1$ resum $\alpha_{s}^{n} \ln ^{n} N$ contributions to all orders in perturbation theory. They can be derived directly by the integral representations eqs. (2.7)-(2.9) by computing the integrals as an expansion in powers of $\alpha_{s}$ at fixed $\alpha_{s} \ln N$, in the large $N$ limit.

Other resummation schemes, which are equivalent to $N$-soft at large $N$, but preserve the analytic structure of fixed-order coefficient functions at finite $N$, have been considered in the context of Higgs production [36, 113, 114] and heavy quark production [115]. The extension of these resummation schemes to DIS and DY, and their application to the determination of PDFs, will be considered elsewhere.

Threshold resummation can be extended to rapidity distributions, see e.g. [52-58]. In this work we follow the approach of ref. [58]. The basic observation is that the resummed partonic rapidity distribution coincides with the rapidity integrated one up to terms which are power-suppressed in the threshold limit. Therefore, in order to obtain the hadron-level resummed rapidity distribution, we have only to modify the parton luminosity. While we refer the Reader to ref. [58] for an explicit derivation, here we limit ourselves to note that the resummed rapidity distribution is constructed in such a way that the integral over rapidity gives back the resummation of the rapidity-integrated cross section.

Finally, we mention that the calculation of hadron-level cross sections and distributions from resummed results in $N$ space requires a prescription because of the presence of a logarithmic branch-cut for real $N>N_{L}$, originating from the Landau pole of the running coupling. As a consequence, the resummed result does not admit an inverse-Mellin transform. Different solutions to this problem exists, such as the Minimal Prescription [116], which consists on a simple modification of the Mellin inversion integral, and the Borel prescription [58, 117-120], which relies on a Borel summation of the divergent series of the order-by-order inverse Mellin transform of the resummed coefficient function. In this paper 
we adopt the Minimal Prescription, but we stress that from a practical point of view, differences between these prescriptions become only relevant at extremely large values $x$ [120], a region where no experimental data is available.

\subsection{Numerical implementation}

In this section we discuss the numerical implementation of the $N$-soft threshold resummation described above. For the PDF fits performed in this work, the processes that we are interested in are DIS (both neutral and charged currents), lepton-pair invariant mass and rapidity distribution for Drell-Yan production, and inclusive top pair production cross section. For DIS and Drell-Yan, we use a new version of the public code ResHiggs, written originally [36] to perform threshold resummation of Higgs inclusive cross section, including several improvements with respect to standard $N$-soft resummation, and later extended [37] to also perform (improved) resummation in the Soft-Collinear Effective Theory formalism. Because of the inclusion of additional processes, the new version of this code changes name from ResHiggs to TROLL, standing for TROLL Resums Only Large-x Logarithms, publicly available at the webpage [121]. To give continuity with the original code ResHiggs, the first version of TROLL is v3.0. For top pair production we use the public code Top++ [7].

The code TROLL is designed to provide only the contribution of the resummation, while the fixed-order calculation is obtained from a separate code (in our case, the same FKgenerator code used for the NNPDF3.0 fits). More specifically, the output of TROLL is $\Delta_{j} K_{\mathrm{N}^{k} \mathrm{LL}}$, defined as the difference between a resummed $K$-factor at $\mathrm{N}^{j} \mathrm{LO}+\mathrm{N}^{k} \mathrm{LL}$ and a fixed-order $K$-factor at $\mathrm{N}^{j} \mathrm{LO}$, such that

$$
\sigma_{\mathrm{N}^{j} \mathrm{LO}+\mathrm{N}^{k} \mathrm{LL}}=\sigma_{\mathrm{N}^{j} \mathrm{LO}}+\sigma_{\mathrm{LO}} \times \Delta_{j} K_{\mathrm{N}^{k} \mathrm{LL}}
$$

where all the cross sections appearing in the above equation are evaluated with a common $\mathrm{N}^{j} \mathrm{LO}+\mathrm{N}^{k} \mathrm{LL}$ PDF set. Internally, $\Delta_{j} K_{\mathrm{N}^{k} \mathrm{LL}}$ is computed by subtracting off the expansion of the resummed coefficient up to $\mathcal{O}\left(\alpha_{s}^{j}\right)$ from the coefficient itself, multiplying this by the parton luminosity, computing the inverse Mellin transform and finally dividing by the parton luminosity.

There are several advantages in using the $\Delta K$-factors defined in eq. (2.11): the fixedorder normalisation is irrelevant, the $K$-factor is much less sensitive to the input PDFs than the cross section itself, and finally, since the resummed contribution has the same kinematic structure as the Born cross section (soft radiation does not change the kinematics), the effect of phase space constraints like kinematic cuts are correctly taken into account if they are applied to the LO cross section in eq. (2.11).

We note that in DIS, Target Mass Corrections (TMCs) at next-to-leading twist are included in the resummation according to the same prescription used in the NNPDF fitting code [88], which amounts to multiplying the Mellin transforms of the partonic coefficient functions by an $N$-dependent factor. No TMCs are included for the fixed-target DY data.

In figure 1 we show the $\Delta K$-factors for the neutral current DIS structure function $F_{2}(x, Q)$, as a function of $x$, for $Q=2 \mathrm{GeV}$ and $Q=30 \mathrm{GeV}$. The plot on the left 

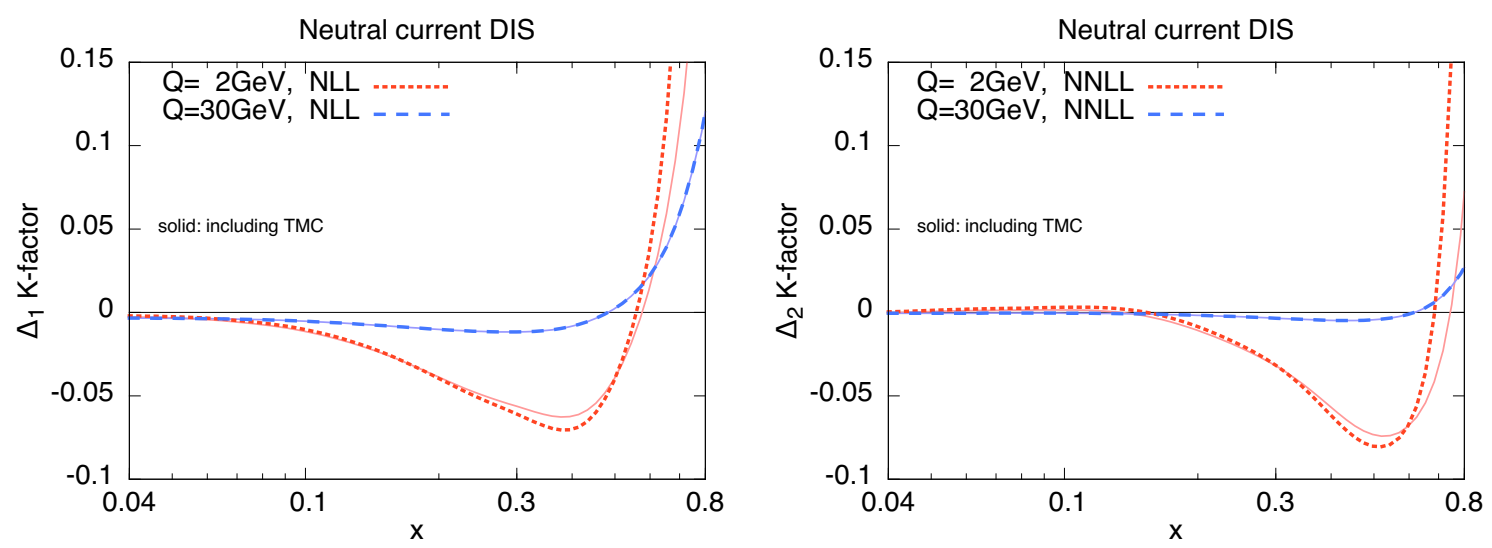

Figure 1. $\Delta K$-factors eq. (2.11) for the neutral current DIS structure function $F_{2}(x, Q)$, as a function of $x$, for $Q=2 \mathrm{GeV}$ and $Q=30 \mathrm{GeV}$. The plot on the left corresponds to $j=1, k=1$ in eq. (2.11), i.e. NLO and NLL, while the one on the right to $j=2, k=2$, i.e. NNLO and NNLL. The effect of adding TMCs is shown as a thin solid line.
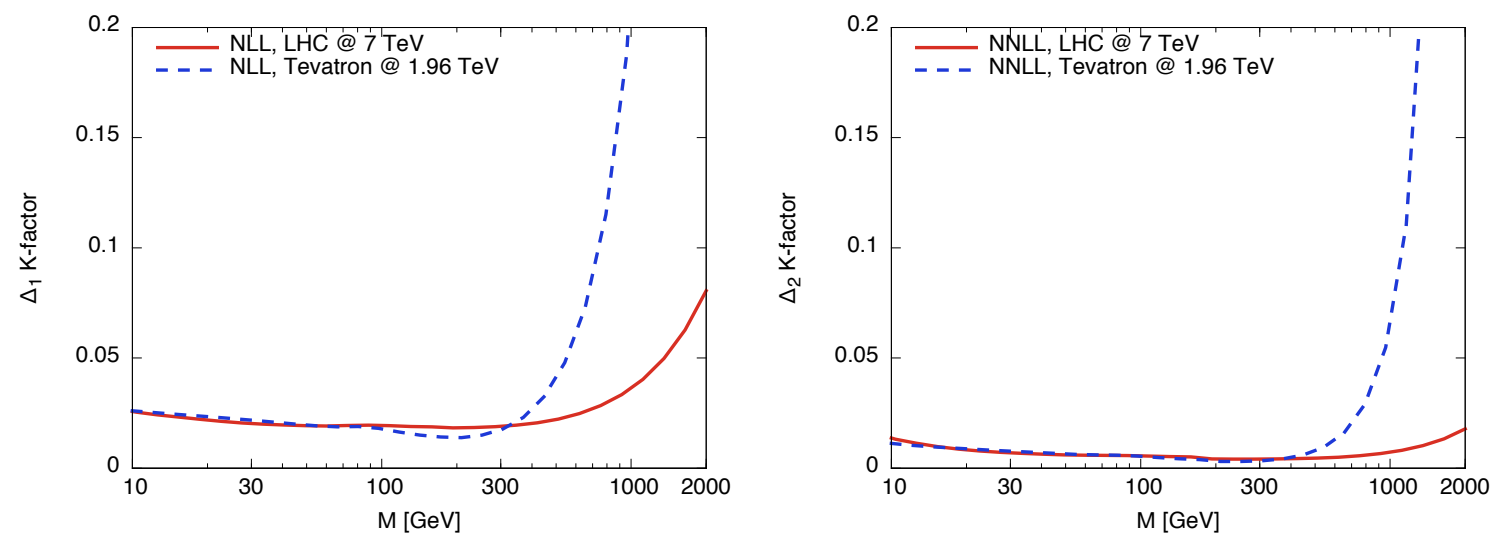

Figure 2. Same as figure 1 for the NLL (left plot) and NNLL (right plot) resummation of neutralcurrent Drell-Yan invariant mass distribution at the Tevatron and at the LHC.

corresponds to $j=1, k=1$ in eq. (2.11), i.e. NLL to be matched to NLO, while the one on the right to $j=2, k=2$, i.e. NNLL to be matched to NNLO. We note that the resummation enhances the cross section at large $x$, while it gives a very small contribution at small $x$, as it should. We also note a dip in the region of intermediate $x$, which is also present in fixed-order calculations [122].

TMC effects are also shown as light shadows to the actual curves: as expected, at large scales they are negligible, while at smaller scales their effect is non-negligible, in particular at large $x$, where they reduce the effect of the resummation. Note that in the definition of $\Delta K$ we use the fact that the same TMCs are already included in the LO cross section, so much of their effect cancels out.

In figure 2 we show the corresponding $\Delta K$-factors this time for Drell-Yan invariant mass distributions, for LHC at $\sqrt{s}=7 \mathrm{TeV}$ and for the Tevatron at $\sqrt{s}=1.96 \mathrm{TeV}$. In figure 3 we also present the results for the lepton pair rapidity distribution in DY processes 

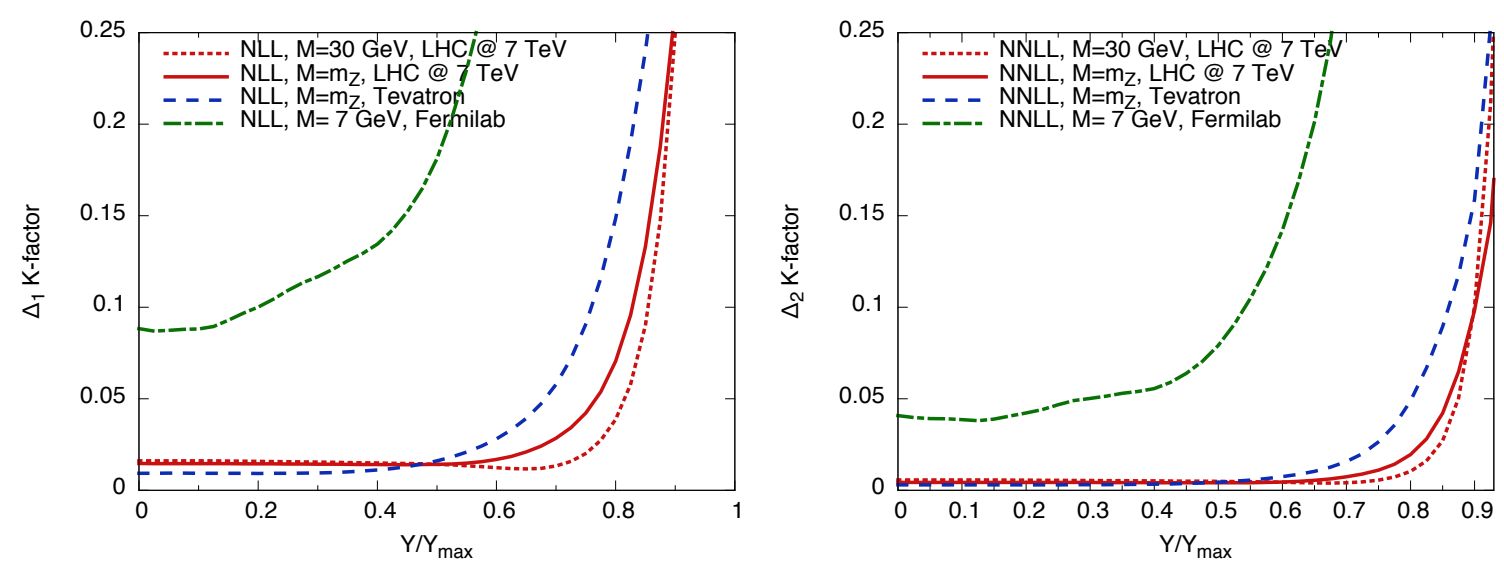

Figure 3. Same as figure 1 for the neutral-current Drell-Yan rapidity distribution, for different experiments and different values of the lepton invariant mass.

\begin{tabular}{|c|cc|}
\hline & LHC $7 \mathrm{TeV}$ & $\mathrm{LHC} 8 \mathrm{TeV}$ \\
\hline$\sigma_{\mathrm{NLO}+\mathrm{NLL}} / \sigma_{\mathrm{NLO}}$ & 1.086 & 1.081 \\
$\sigma_{\mathrm{NNLO}+\mathrm{NNLL}} / \sigma_{\mathrm{NNLO}}$ & 1.031 & 1.029 \\
$\sigma_{\mathrm{NNLO}} / \sigma_{\mathrm{NLO}}$ & 1.123 & 1.122 \\
\hline
\end{tabular}

Table 1. $K$-factors for $t \bar{t}$ production at $\mathrm{LHC}$ at 7 and $8 \mathrm{TeV}$. The first line is obtained using NLO PDFs, while the second and third lines are obtained with NNLO PDFs (in all cases, both numerator and denominator are computed with the same PDFs).

as a function of $Y / Y_{\max }$, with $Y_{\max }=\frac{1}{2} \ln \left(s / M^{2}\right)$ being the maximum rapidity of the lepton pairs allowed by kinematics. The experiments cover different kinematic regimes: close to threshold (Fermilab's fixed-target Drell-Yan experiments at $M=7 \mathrm{GeV}$ ), an intermediate region (Tevatron and LHC at the $Z$ pole) and away from threshold (low mass DY at LHC). We note that threshold resummation always gives a significant correction at large rapidities. For the fixed-target kinematics and $M=7 \mathrm{GeV}$, the effect of threshold resummation is important even at central rapidities.

Finally, in table 1 we collect the $K$-factors for $t \bar{t}$ production obtained using Top++. In this case we provide directly the $K$-factors for the $(\mathrm{N}) \mathrm{NLO}+(\mathrm{N}) \mathrm{NLL}$ over (N)NLO cross sections, with both numerator and denominator computed with the same (N)NLO PDFs. We can see from the table that the impact of the resummation is non-negligible, and in fact rather important especially at NLO+NLL, where the correction is about $9 \%$ of the fixed-order NLO result, comparable to the NNLO correction. Even at NNLO+NNLL, the effect of resummation is comparable to other theory uncertainties like the values of $\alpha_{s}\left(m_{Z}^{2}\right)$ or of the top quark mass [123].

\section{Settings of the resummed PDF fit}

In this section we present the settings used in the resummed PDF fits. These are constructed as variant of the recent NNPDF3.0 global fits [97]: they use exactly the same 
fitting methodology, the same input parameters (strong coupling, heavy quark masses, etc.), and the same fixed-order theoretical calculations. The experimental dataset is also similar except that some specific processes have been excluded.

In this section, we first review the experimental data that can be consistently included in a threshold resummed global PDF analysis, and then we explain the procedure used to construct the resummed $K$-factors that will be used to include threshold resummation in the NNPDF fits. We also show the resulting resummed $K$-factors for a representative subset of the experiments used in the fit.

\subsection{Experimental data}

In a PDF fit with threshold resummation, as compared to fixed-order fits, some datasets cannot be included since for these processes threshold resummation is either unknown or not currently available in a format that can be used in a fit. In particular, when compared to NNPDF3.0, in the present resummed fit we include all the neutral and charged current DIS data, neutral current DY production and top quark pair production data. However we exclude the DY charged current datasets, for which data is provided in terms of the lepton kinematics, and the construction of resummed expressions is more involved, and inclusive jet production for the reasons discussed in section 2.1.

In table 2 we list all the datasets used in the NNPDF3.0 NLO and NNLO global analysis, and indicate whether or not they are now included in the NLL and NNLL resummed NNPDF3.0 fits. For each dataset we also display the corresponding measured observable, and the relevant publication. A more complete description of each of these datasets, as well as of their impact in terms of PDF constraints in the global fit, can be found in [97].

From table 2, we infer that, when compared to the global fit, the resummed fits lose experimental constraints on the medium and large- $x$ gluon (due to the exclusion of the jet data) and on the quark-flavor separation (due to the exclusion of the $W$ data). Still, given that we include in the resummed fit more than 3000 data points, the loss of accuracy due to the exclusion of these datasets is not dramatic, as we will show in section 4 . In future studies, we aim to include the missing processes once the corresponding resummed calculations become available.

The kinematic cuts applied in the present fits closely follow the ones of the NNPDF3.0 fixed-order analysis. In particular, a cut on the final-state invariant mass of DIS data $W^{2} \geq 12.5 \mathrm{GeV}^{2}$ is applied, in order to reduce the dependence on higher-twists at large $x$. It would be interesting to loosen this cut in future analyses, in order to test the stability of the leading-twist PDF determination once the large- $x$ resummation is included; this might also allow us to include additional large- $x$, low- $Q^{2}$ DIS measurements, such as for example JLAB data [164].

In addition, a stability analysis of the calculations for neutral-current Drell-Yan production indicates that our results, even when supplemented with resummation, become unstable for data points too close to the production threshold, either because the invariant mass $M_{l l}$ of the Drell-Yan pairs is too large, or because the rapidity $Y$ is too close to the kinematic boundary $Y_{\max }$. Therefore, we have supplemented the NNPDF3.0 kinematic 


\begin{tabular}{|c|c|c|c|c|}
\hline Experiment & Observable & Ref. & $\begin{array}{l}\text { NNPDF3.0 global } \\
(\mathrm{N}) \mathrm{NLO}\end{array}$ & $\begin{array}{c}\text { NNPDF3.0 DIS+DY+top } \\
(\mathrm{N}) \mathrm{NLO}[+(\mathrm{N}) \mathrm{NLL}]\end{array}$ \\
\hline $\mathrm{NMC}$ & $\sigma_{\mathrm{dis}}^{\mathrm{NC}}, F_{2}^{d} / F_{2}^{p}$ & {$[124,125]$} & Yes & Yes \\
\hline BCDMS & $F_{2}^{d}, F_{2}^{p}$ & {$[126,127]$} & Yes & Yes \\
\hline SLAC & $F_{2}^{d}, F_{2}^{p}$ & {$[128]$} & Yes & Yes \\
\hline CHORUS & $\sigma_{\nu N}^{\mathrm{CC}}$ & {$[129]$} & Yes & Yes \\
\hline $\mathrm{NuTeV}$ & $\sigma_{\nu N}^{\mathrm{CC}, \text { charm }}$ & {$[130]$} & Yes & Yes \\
\hline HERA-I & $\sigma_{\text {dis }}^{\mathrm{NC}}, \sigma_{\text {dis }}^{\mathrm{CC}}$ & {$[131]$} & Yes & Yes \\
\hline ZEUS HERA-II & $\sigma_{\text {dis }}^{\mathrm{NC}}, \sigma_{\text {dis }}^{\mathrm{CC}}$ & {$[132-135]$} & Yes & Yes \\
\hline H1 HERA-II & $\sigma_{\text {dis }}^{\mathrm{NC}}, \sigma_{\text {dis }}^{\mathrm{CC}}$ & {$[136,137]$} & Yes & Yes \\
\hline HERA charm & $\sigma_{\text {dis }}^{\mathrm{NC}, \text { charm }}$ & {$[138]$} & Yes & Yes \\
\hline DY E866 & $\sigma_{\mathrm{DY}, \mathrm{p}}^{\mathrm{NC}}, \sigma_{\mathrm{DY}, \mathrm{d}}^{\mathrm{NC}} / \sigma_{\mathrm{DY}, \mathrm{p}}^{\mathrm{NC}}$ & {$[139-141]$} & Yes & Yes \\
\hline DY E605 & $\sigma_{\mathrm{DY}, \mathrm{p}}^{\mathrm{NC}}$ & {$[142]$} & Yes & Yes \\
\hline CDF $Z$ rap & $\sigma_{\mathrm{DY}, \mathrm{p}}^{\mathrm{NC}}$ & {$[143]$} & Yes & Yes \\
\hline CDF Run-II $k_{t}$ jets & $\sigma_{\text {jet }}$ & {$[144]$} & Yes & No \\
\hline D0 $Z$ rap & $\sigma_{\mathrm{DY}, \mathrm{p}}^{\mathrm{NC}}$ & {$[145]$} & Yes & Yes \\
\hline ATLAS $Z 2010$ & $\sigma_{\mathrm{DY}, \mathrm{p}}^{\mathrm{NC}}$ & {$[146]$} & Yes & Yes \\
\hline ATLAS $W 2010$ & $\sigma_{\mathrm{DY}, \mathrm{p}}^{\mathrm{CC}}$ & {$[146]$} & Yes & No \\
\hline ATLAS $7 \mathrm{TeV}$ jets 2010 & $\sigma_{\text {jet }}$ & {$[147]$} & Yes & No \\
\hline ATLAS $2.76 \mathrm{TeV}$ jets & $\sigma_{\text {jet }}$ & {$[148]$} & Yes & No \\
\hline ATLAS high-mass DY & $\sigma_{\mathrm{DY}, \mathrm{p}}^{\mathrm{NC}}$ & {$[149]$} & Yes & Yes \\
\hline ATLAS $W p_{T}$ & $\sigma_{\mathrm{DY}, \mathrm{p}}^{\mathrm{CC}}$ & {$[150]$} & Yes & No \\
\hline CMS $W$ electron asy & $\sigma_{\mathrm{DY}, \mathrm{p}}^{\mathrm{CC}}$ & {$[151]$} & Yes & No \\
\hline CMS $W$ muon asy & $\sigma_{\mathrm{DY}, \mathrm{p}}^{\mathrm{CC}}$ & {$[152]$} & Yes & No \\
\hline CMS jets 2011 & $\sigma_{\mathrm{jet}}$ & {$[153]$} & Yes & No \\
\hline CMS $W+c$ total & $\sigma_{\mathrm{DY}, \mathrm{p}}^{\mathrm{NC}, \text { charm }}$ & {$[154]$} & Yes & No \\
\hline CMS 2D DY 2011 & $\sigma_{\mathrm{DY}, \mathrm{p}}^{\mathrm{NC}}$ & {$[155]$} & Yes & Yes \\
\hline LHCb $W$ rapidity & $\sigma_{\mathrm{DY}, \mathrm{p}}^{\mathrm{CC}}$ & {$[156]$} & Yes & No \\
\hline LHCb $Z$ rapidity & $\sigma_{\mathrm{DY}, \mathrm{p}}^{\mathrm{NC}}$ & {$[157]$} & Yes & Yes \\
\hline ATLAS CMS top prod & $\sigma(t \bar{t})$ & {$[158-163]$} & Yes & Yes \\
\hline
\end{tabular}

Table 2. List of all the experiments that were used in the NNPDF3.0 global analysis, and whether or not they are now included in the present (N)NLL resummed fits (and in the corresponding baseline fixed-order fits). For each dataset we also provide the type of cross section that has been measured and the corresponding publication references.

cuts with two additional cuts for the fixed-target Drell-Yan experiments, as summarised in table 3. For the collider Drell-Yan data, the cuts are the same as in NNPDF3.0.

It is useful to quantify which experiments determine the behaviour of the large- $x$ PDFs in the global analysis. In figure 4 we compare the relative PDF uncertainties in variants of the NNPDF3.0 NLO fit based on different input datasets: HERA-only, no LHC data, no jet data, a DIS+DY+top fit (our baseline for the resummed fits) and the global fit. We focus in the large- $x$ region for $Q^{2}=10^{4} \mathrm{GeV}$, a typical scale for LHC phenomenology. We show the gluon, the total quark singlet, the total valence and the $\bar{u}$ quark PDFs. From this comparison we see that the PDF that at large- $x$ is most dependent on the choice of 


\begin{tabular}{|c|c|}
\hline Experiment & Kinematic cuts \\
\hline DIS & $Q^{2} \geq Q_{\min }^{2}=3.5 \mathrm{GeV}^{2}$ \\
& $W^{2} \geq W_{\min }^{2}=12.5 \mathrm{GeV}^{2}$ \\
\hline Fixed target Drell-Yan & $\tau \leq 0.08$ \\
& $|Y| / Y_{\max } \leq 0.663$ \\
\hline
\end{tabular}

Table 3. Kinematic cuts applied to the DIS and fixed-target Drell-Yan data in the baseline and resummed fits. For the collider Drell-Yan data, the cuts are the same as in NNPDF3.0.
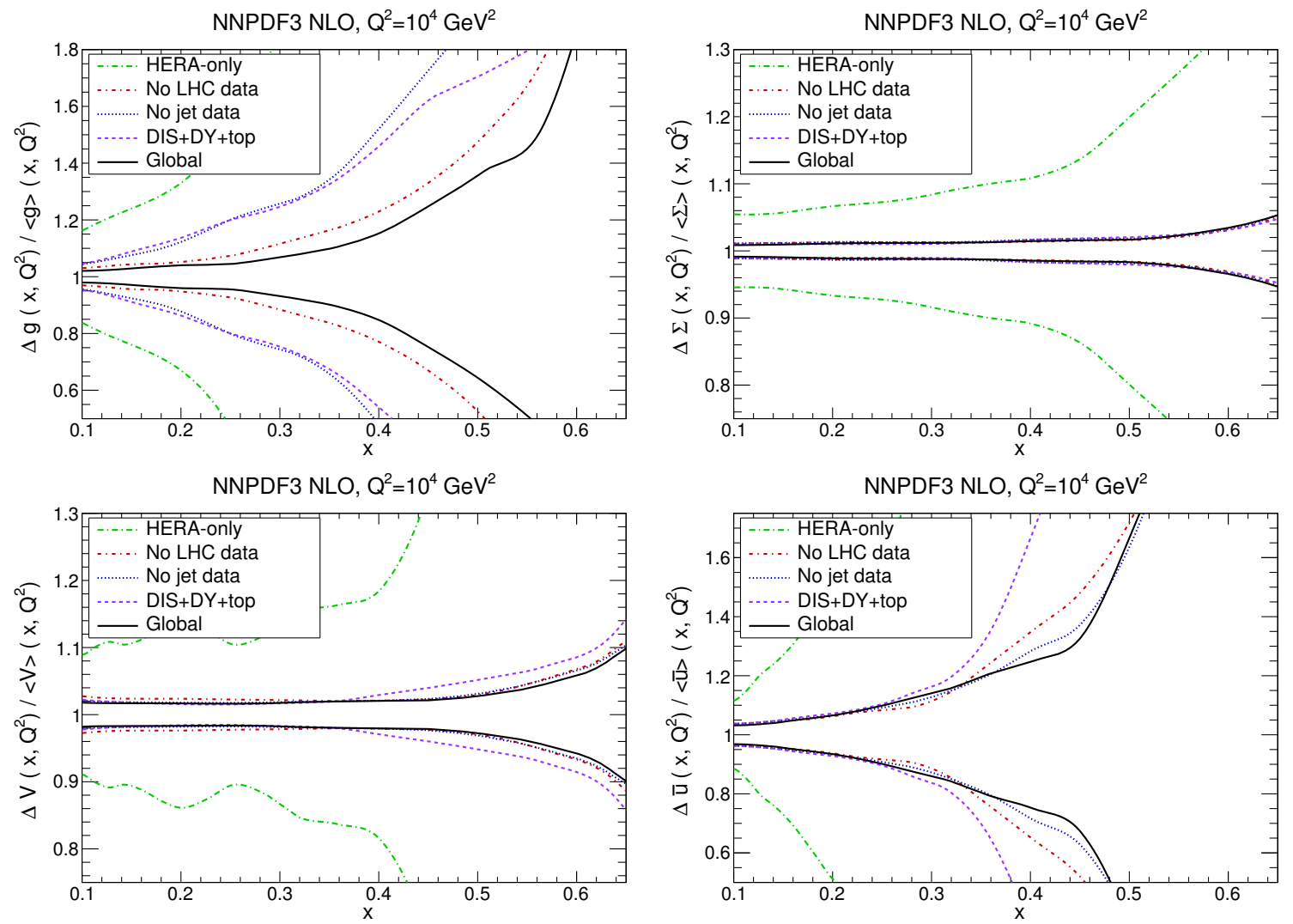

Figure 4. Comparison of the relative PDF uncertainty at large- $x$ between NNPDF3.0 NLO fits based on different input datasets. We show the results for the gluon, total quark singlet, total valence and $\bar{u}$ quark PDFs, at a typical LHC scale of $Q^{2}=10^{4} \mathrm{GeV}^{2}$. The fits shown are the HERA-only, no LHC data, no jet data, DIS+DY+top (our baseline for the resummed fits) and finally the global fit.

input dataset is the gluon. For the total valence and singlet quark PDFs, the bulk of the constraints are provided by the DIS and fixed target Drell-Yan data, which are common datasets in all these fits (except for the HERA-only fit). For the sea quarks, in this case the $\bar{u}$ quark, the information on both jet data and LHC data are necessary to achieve the best possible accuracy. The baseline DIS+DY+top fit is slightly less accurate at large- $x$ for the quark flavor separation due to the missing charged-current Drell-Yan data. 


\subsection{Calculation of resummed $K$-factors}

As mentioned in the introduction, in the $\overline{\mathrm{MS}}$ scheme all the effects of threshold resummation are encoded in the partonic cross sections, and thus parton evolution is the same as in fixed-order calculations. Therefore, apart from the modification of the hard-scattering cross sections, all theoretical settings in the resummed fit will be the same as those of the NNPDF3.0 fixed-order analysis, including the use of the FONLL general-mass VFN scheme [165], the values of the heavy quark masses, and so on. We will produce results for single a value of the strong coupling, $\alpha_{s}\left(m_{Z}^{2}\right)=0.118$.

As discussed in ref. [97], the NNPDF3.0 global analysis for hadronic observables always uses fast NLO calculations [166-168] supplemented with NNLO/NLO $K$-factors when required. These are defined as the ratio of the NNLO over the NLO bin-by-bin cross sections, using a common PDF luminosity computed with a NNLO PDF set. For the resummed fits, we follow exactly the same procedure: we include the effect of resummation supplementing the fixed-order computation with a $K$-factor. Since the $K$-factor is computed externally using a fixed set of PDFs, the fit is re-iterated several times, recomputing each time the resummed $K$-factor using as input PDFs those obtained from the previous iteration.

As discussed in section 2.2, the resummed contributions for DIS and DY processes is obtained using the program TROLL in the form of $\Delta K$-factors, eq. (2.11), and hence must be converted into actual $K$-factors. For DIS cross sections, since the NNLO calculation is implemented exactly in the NNPDF fitting code, this is done according to

$$
K_{\mathrm{DIS}}^{\mathrm{N}^{k} \mathrm{LO}+\mathrm{N}^{k} \mathrm{LL}} \equiv \frac{\sigma^{\mathrm{N}^{k} \mathrm{LO}+\mathrm{N}^{k} \mathrm{LL}}}{\sigma^{\mathrm{N}^{k} \mathrm{LO}}}=1+\Delta_{k} K_{\mathrm{N}^{k} \mathrm{LL}} \cdot \frac{\sigma^{\mathrm{LO}}}{\sigma^{\mathrm{N}^{k} \mathrm{LO}}},
$$

with $k=1,2$ for NLO+NLL and NNLO+NNLL respectively. For hadronic processes we use a similar expression, but (at NNLO) also including the NNLO/NLO $K$-factor,

$$
\begin{aligned}
K_{\mathrm{hadr}}^{\mathrm{NLO}+\mathrm{NLL}} & \equiv \frac{\sigma^{\mathrm{NLO}+\mathrm{NLL}}}{\sigma^{\mathrm{NLO}}}=1+\Delta_{1} K_{\mathrm{NLL}} \cdot \frac{\sigma^{\mathrm{LO}}}{\sigma^{\mathrm{NLO}}}, \\
K_{\mathrm{hadr}}^{\mathrm{NNLO}+\mathrm{NNLL}} & \equiv \frac{\sigma^{\mathrm{NNLO}+\mathrm{NNLL}}}{\sigma^{\mathrm{NLO}}}=K^{\mathrm{NNLO}}+\Delta_{2} K_{\mathrm{NNLL}} \cdot \frac{\sigma^{\mathrm{LO}}}{\sigma^{\mathrm{NLO}}},
\end{aligned}
$$

where $K^{\mathrm{NNLO}}=\sigma^{\mathrm{NNLO}} / \sigma^{\mathrm{NLO}}$. In the above expressions, all contributions are meant to be computed with the same $\mathrm{N}^{k} \mathrm{LO}+\mathrm{N}^{k} \mathrm{LL}$ PDF set. For the leading-order cross section $\sigma^{\mathrm{LO}}$, dedicated FK tables with LO coefficient functions but NLO and NNLO PDF evolution have been produced using the APFEL program [169], validated with the same FKgenerator internal code used in the NNPDF3.0 fits.

In principle, all these contributions should be recomputed at each iteration of the fit; in practice, the computation of $K^{\mathrm{NNLO}}$ is time consuming, so for this contribution we use a fixed value. Specifically, in the present work, these fixed-order NNLO/NLO $K$-factors are the same as in the NNPDF3.0 fits, with the exception of those for fixed-target Drell-Yan experiments, which have been recomputed using Vrap [170] with the NNPDF3.0 global PDF set as input.

For the computation of the resummed $K$-factors eq. (3.1)-(3.3), we find that two iterations of the fit are enough to reach a satisfactory convergence, meaning that these 

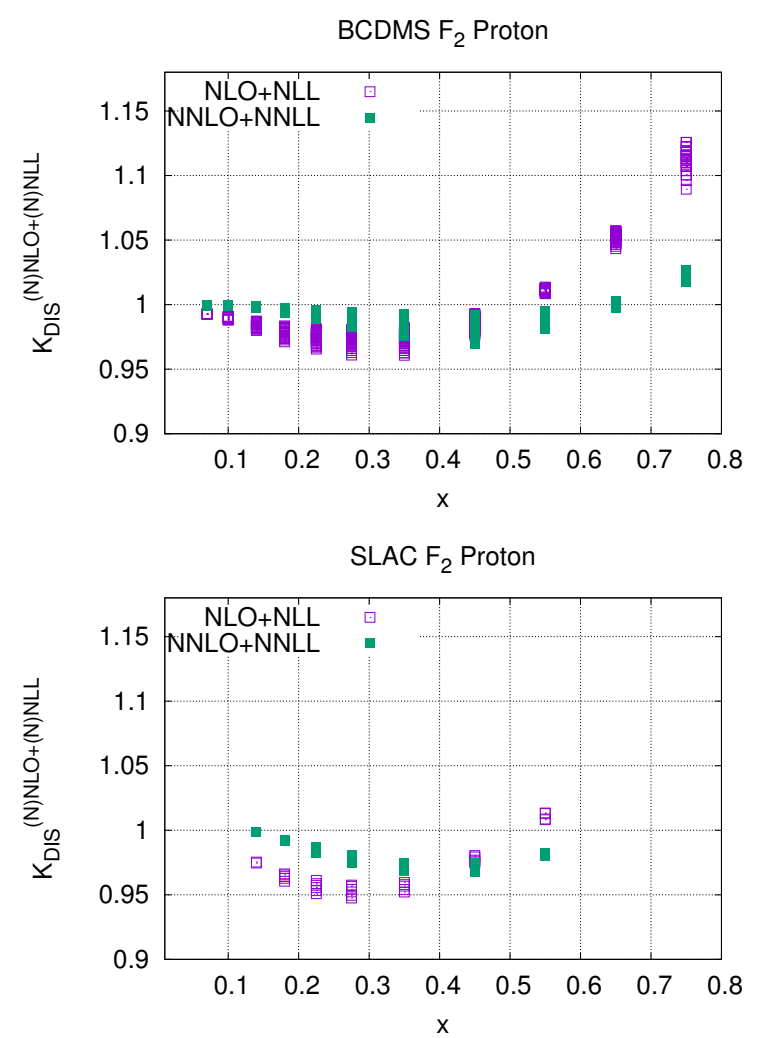

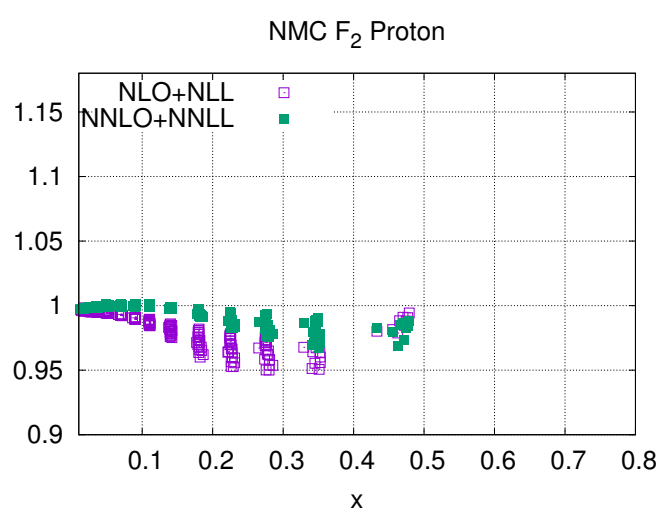

CHORUS Neutrino DIS

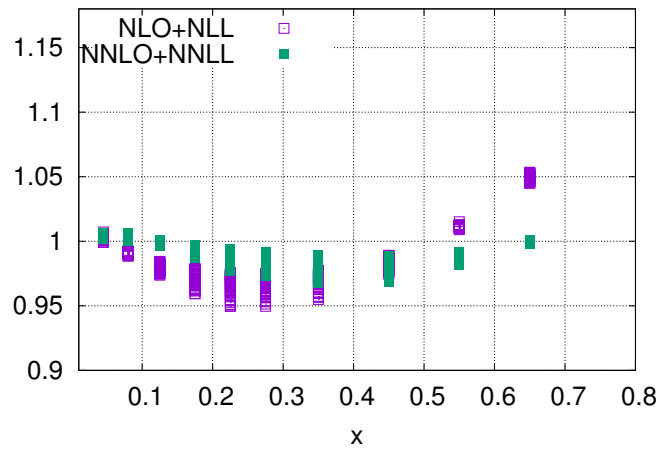

Figure 5. The resummed $K$-factors for DIS, eq. (3.1), for a representative subset of the experiments included in the resummed fit: BCDMS $F_{2}^{p}$, NMC $\sigma_{\mathrm{NC}}^{p}$, SLAC $F_{2}^{p}$ and CHORUS $\sigma_{\nu N}$. We show both the results corresponding to NLL and to NNLL resummation. The DIS kinematics $\left(x, Q^{2}, y\right)$ is that of the corresponding experimental data included in the fit, plotted just as a function of $x$, so for each value of $x$ there are measurements at different values of $Q^{2}$ and $y$.

$K$-factors are essentially unchanged if we use resummed PDFs from the last or from the next-to-last iteration of the fit.

It is now interesting to illustrate the effect of the (N)NLL resummation for some of the datasets used in the present resummed PDF fit. To this purpose, we plot the DIS, eq. (3.1), and hadronic, eqs. (3.2) and (3.3), resummed $K$-factors for representative experimental datasets with exactly the same kinematics as for the data points to be used in the fit. In these calculations, we have consistently used the NNPDF3.0 DIS+DY+top NLO+NLL and NNLO+NNLL PDF sets discussed in the next section, with $\alpha_{s}\left(m_{Z}^{2}\right)=0.118$, in both the fixed-order and resummed cross sections. To isolate the effect of the resummation, in these comparison plots we will factor out $K^{\mathrm{NNLO}}$ from the hadronic NNLO+NNLL resummed $K$-factor eq. (3.3). Note that in these plots we will only include those data points that satisfy the kinematic cuts imposed in the fit, summarised in table 3.

The results for the DIS case are shown in figure 5. For each experiment, we show both the results corresponding to NLL and to NNLL resummation. The DIS kinematics $\left(x, Q^{2}, y\right)$ is that of the associated experimental measurements, so for each value of $x$ there are measurements at different values of $Q^{2}$ and $y$. We do not show the results for any of the 

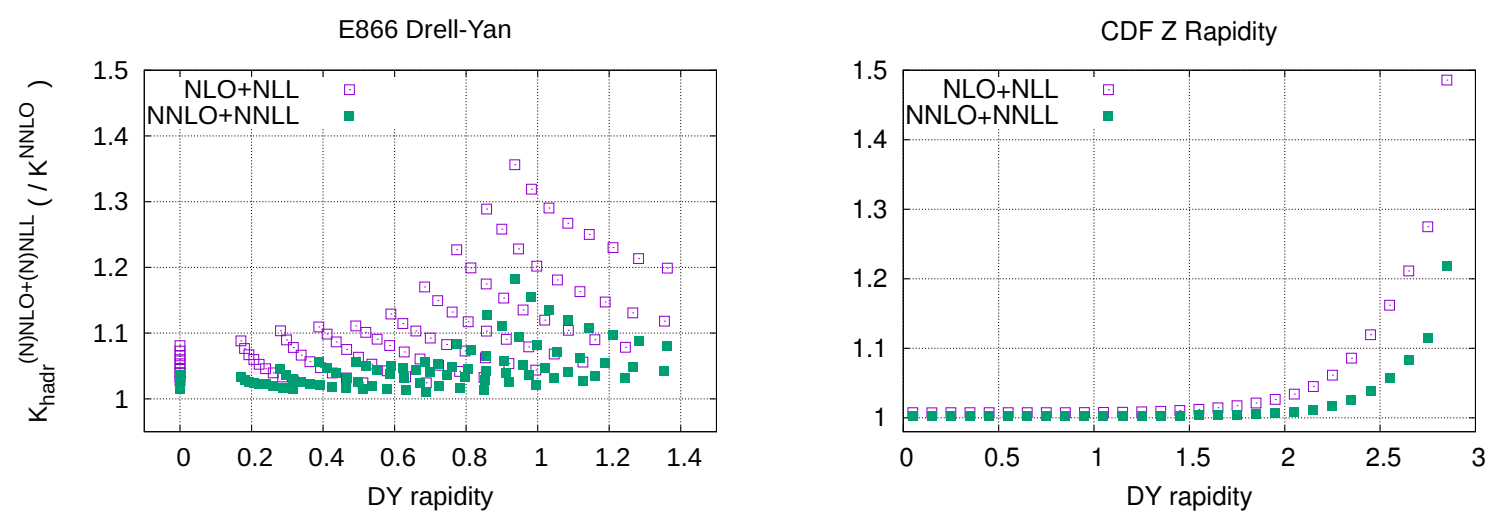

CMS Drell-Yan 2D 2011

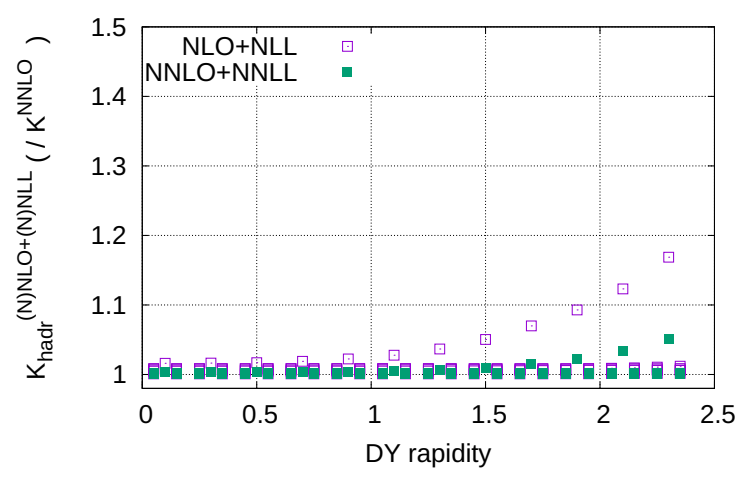

LHCb Z rapidity 2011

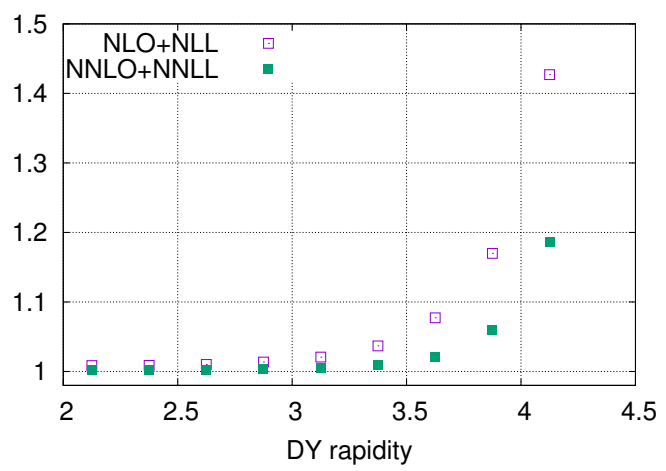

Figure 6. Same as figure 5 for selected Drell-Yan experimental datasets included in the fit: the E866 $p p$ neutral Drell-Yan cross sections, the CDF $Z$ rapidity distribution, the CMS double-differential Drell-Yan distribution at $7 \mathrm{TeV}$ and the LHCb $Z \rightarrow \mu \mu$ rapidity distribution. The resummed $K$ factors are now those defined in eqs. (3.2) and (3.3), but to isolate the effect of resummation from that of the fixed-order NNLO corrections, in the NNLL case we divide eq. (3.3) by $K^{\mathrm{NNLO}}$. In the two left plots data points differ by the values of the rapidity and the invariant mass of the pair, but only the dependence on the rapidity is shown.

HERA datasets, for which the effect of the resummation turns out to be negligible since the data is either at small- $x$ or at high scales. As expected, the impact of the resummation is only relevant at large $x$, and of course the impact of the resummation decreases when more fixed perturbative orders are included in the calculation.

From the results of figure 5 we see that the effect of threshold resummation is most important for the BCDMS data, while it is milder for the other experiments. Effects of NLL resummation reach up to $15 \%$ at the highest values of $x$ available, which is reduced to up to a few percent for NNLL resummation (since part of the effects at NLL are now included in the fixed NNLO calculation). Note also that the cut in $W^{2}$ removes most of the large- $x$ SLAC data, where resummation effects are very large. We also note that the effect of resummation is comparable to the experimental uncertainties, and thus we should expect to see an impact on the resulting large- $x$ parton distributions.

The corresponding results for the resummed $K$-factors eq. (3.3) for representative neutral-current DY experiments are collected in figure 6. As in the case of DIS, except 
for $Z$ peak measurements, for each DY rapidity $Y$ value there are various data points at different invariant mass $M$. We show the Drell-Yan E866 $p p$ cross sections, the CDF $Z$ rapidity distribution, the CMS double-differential Drell-Yan distribution at $7 \mathrm{TeV}$ and the LHCb $Z \rightarrow \mu \mu$ rapidity distribution.

From figure 6 we verify the expectation that the impact of resummation is rather more important at NLO+NLL than at NNLO+NNLL, and that it grows with the di-lepton rapidity (since in this case the kinematic threshold is approached). For those collider measurements differential in rapidity, the effect of (N)NLL resummation can be as large as $50 \%(20 \%)$ at the highest rapidities. For the fixed-target DY experiments the effect of resummation is substantial even at NNLL, since in this case many data points have kinematics close to threshold. For example, for the E866 pp dataset, the effect of the resummation results is an enhancement of the cross section that can be as large as $35 \%$ at NLL, and $20 \%$ at NNLL.

Following this discussion on the settings used to produce the threshold resummed fits, in the the next section we turn to explore the actual effects that the inclusion of resummed calculations have on the NNPDF3.0 PDFs.

\section{Parton distributions with threshold resummation}

In this section we discuss the results of the NNPDF3.0 fits with threshold resummation. One important difference of the resummed fits as compared to the NNPDF3.0 global fits is that the dataset is different, because we leave out the inclusive jet and $W$ production data, as discussed in section 3. Therefore, first we quantify the information loss due to the reduced dataset by comparing the global NNPDF3.0 fits and the reduced dataset fits obtained with fixed-order matrix elements (henceforth denoted as the baseline fits).

Having established this, we move to quantify the impact of threshold resummation on the fit quality and the resulting PDFs, by comparing fits at NLO and NLO+NLL first, and then at NNLO and NNLO+NNLL. This is done both for DIS-only fits and for DIS+DY+top fits, and the comparison is performed both at the level of PDFs and of $\chi^{2}$. Finally, we assess the impact of threshold resummation at the level of partonic luminosities.

The phenomenological implications of the resummed PDFs for LHC applications will be discussed in the next section.

\subsection{Baseline fixed-order fits}

First of all, we compare the baseline fixed-order fits with the NNPDF3.0 global sets. As mentioned in section 3 , in the fixed-order baseline fits all settings are identical to those of NNPDF3.0 with the only difference of the use of a reduced dataset, see table 2 . Therefore, we expect the two fits to be consistent, with the baseline fit affected by larger PDF uncertainties due to the reduced dataset. For simplicity, we restrict these comparisons to NLO, since the impact of the reduced dataset is roughly independent of the perturbative order.

We have produced two baseline fits: one with the all the data marked in the last column of table 2, and the other with only DIS-data included. In figure 7 we compare the NNPDF3.0 NLO DIS-only and DIS+DY+top set with $\alpha_{s}\left(m_{Z}^{2}\right)=0.118$, with the 

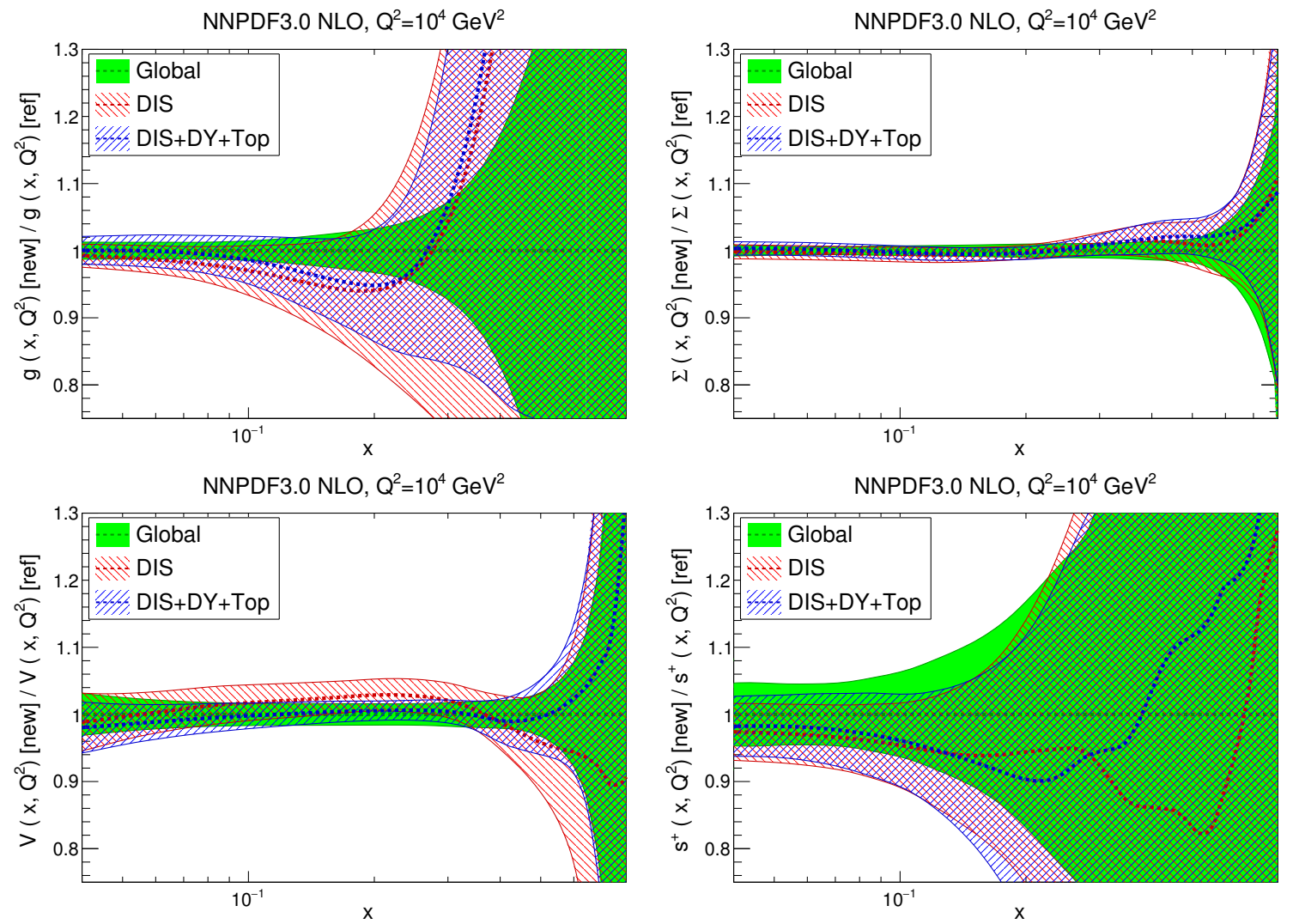

Figure 7. Comparison of the fixed-order NNPDF3.0 NLO fits based on different datasets: global, DIS-only and DIS+DY+top, for $\alpha_{s}\left(m_{Z}^{2}\right)=0.118$, at a typical LHC scale of $Q^{2}=10^{4} \mathrm{GeV}^{2}$. Results are normalised to the central prediction of the NNPDF3.0 NLO global fit. From left to right and from top to bottom, we show the gluon, the total quark singlet, the total valence PDF and the total strangeness.

corresponding global set. ${ }^{3}$ In both cases we use $N_{\text {rep }}=100$ replicas, and the comparison is performed at a typical LHC scale $Q=100 \mathrm{GeV}$. Results are normalised to the central prediction of the global fit. From left to right and from top to bottom, we show the gluon, the total quark singlet, the total valence PDF and the total strangeness.

As we can see, there is a reasonable agreement for most of the PDF flavours and of momentum fraction $x$ between the three fits, with as expected larger PDF uncertainties in the DIS-only and DIS+DY+top fits due to the reduced dataset. The DIS-only fit is relatively close to the global fit for the large- $x$ quarks, since these are well constrained by the DIS fixed-target data. On the other hand, the DIS-only fit is affected by rather larger uncertainties as compared to the global fit for the gluon (due to the missing jet data) and for the total valence (due to the missing Drell-Yan data that constrains flavor separation). In any case, the DIS-only and the global fit are always consistent at the one-sigma level.

\footnotetext{
${ }^{3}$ In the rest of this section, we concentrate only on the large- $x$ region of the PDFs, since as we will show the effects of threshold resummation are negligible at medium and small- $x$.
} 


\begin{tabular}{|c|c|c|c|c|}
\hline Experiment & \multicolumn{4}{|c|}{ NNPDF3.0 DIS-only } \\
\hline & NLO & NNLO & NLO+NLL & NNLO+NNLL \\
\hline NMC & 1.36 & 1.39 & 1.36 & 1.32 \\
SLAC & 1.12 & 1.15 & 1.02 & 1.04 \\
BCDMS & 1.19 & 1.20 & 1.21 & 1.22 \\
CHORUS & 1.10 & 1.05 & 1.09 & 1.07 \\
NuTeV & 0.52 & 0.46 & 0.55 & 0.51 \\
\hline HERA-I & 1.07 & 1.13 & 1.06 & 1.07 \\
ZEUS HERA-II & 1.40 & 1.42 & 1.42 & 1.43 \\
H1 HERA-II & 1.67 & 1.79 & 1.68 & 1.74 \\
HERA charm & 1.28 & 1.29 & 1.29 & 1.24 \\
\hline Total & 1.237 & 1.257 & 1.237 & 1.242 \\
\hline
\end{tabular}

Table 4. The $\chi^{2}$ per data point for all experiments included in the DIS-only threshold resummed fits, at NLO and NNLO, compared with their resummed counterparts.

Concerning the DIS+DY+top fit, for quark PDFs (singlet, valence and strangeness) the results of the DIS+DY+top fit are quite close to the global fit. Therefore, we can conclude that for quark-initiated processes, calculations done with the DIS+DY+top fits are essentially equivalent to those performed using the global PDFs. The only differences are as expected related to the gluon PDF, where the missing inclusive jet data cause a substantial increase in the large- $x$ gluon PDF uncertainties compared to the global fit. Note however that in the resummed DIS+DY+top fit a handle on the large- $x$ gluon is still provided by the total top-quark pair production cross section [123, 171].

After having established the impact of the reduced datasets on the baseline fits that will be used for the resummation, in the following we concentrate in quantifying the impact of resummation for fits based on a common dataset, first for the DIS-only fits, and then for the DIS+DY+top fits.

\subsection{DIS-only resummed PDFs}

Now we present the results of the resummed fits. We begin with the DIS-only fits, and compare the baseline NLO and NNLO fixed-order with the corresponding NLO+NLL and NNLO+NNLL threshold resummed fits.

In table 4 we provide the $\chi^{2}$ per data point for all experiments included in the DISonly threshold resummed fits, at NLO+NLL and NNLO+NNLL, to be compared with their unresummed counterparts. ${ }^{4}$ From table 4 we see that, as expected, the impact of resummation is moderate and restricted to the fixed-target DIS experiments. In the case of SLAC, there is a clear improvement in the $\chi^{2}$ due to the inclusion of threshold resummation, both at NLO and at NNLO. For other experiments the change in $\chi^{2}$ is not significant, meaning that the small effect of threshold resummation can be absorbed in the fitted PDFs.

\footnotetext{
${ }^{4}$ As in NNPDF3.0, the present fits used the $t_{0}$ definition of the covariance matrix for the $\chi^{2}$ minimisation, but then use the experimental definition to assess the consistency between theory and data. See [2, 172] for the explanation of the different definitions of the $\chi^{2}$ estimators.
} 

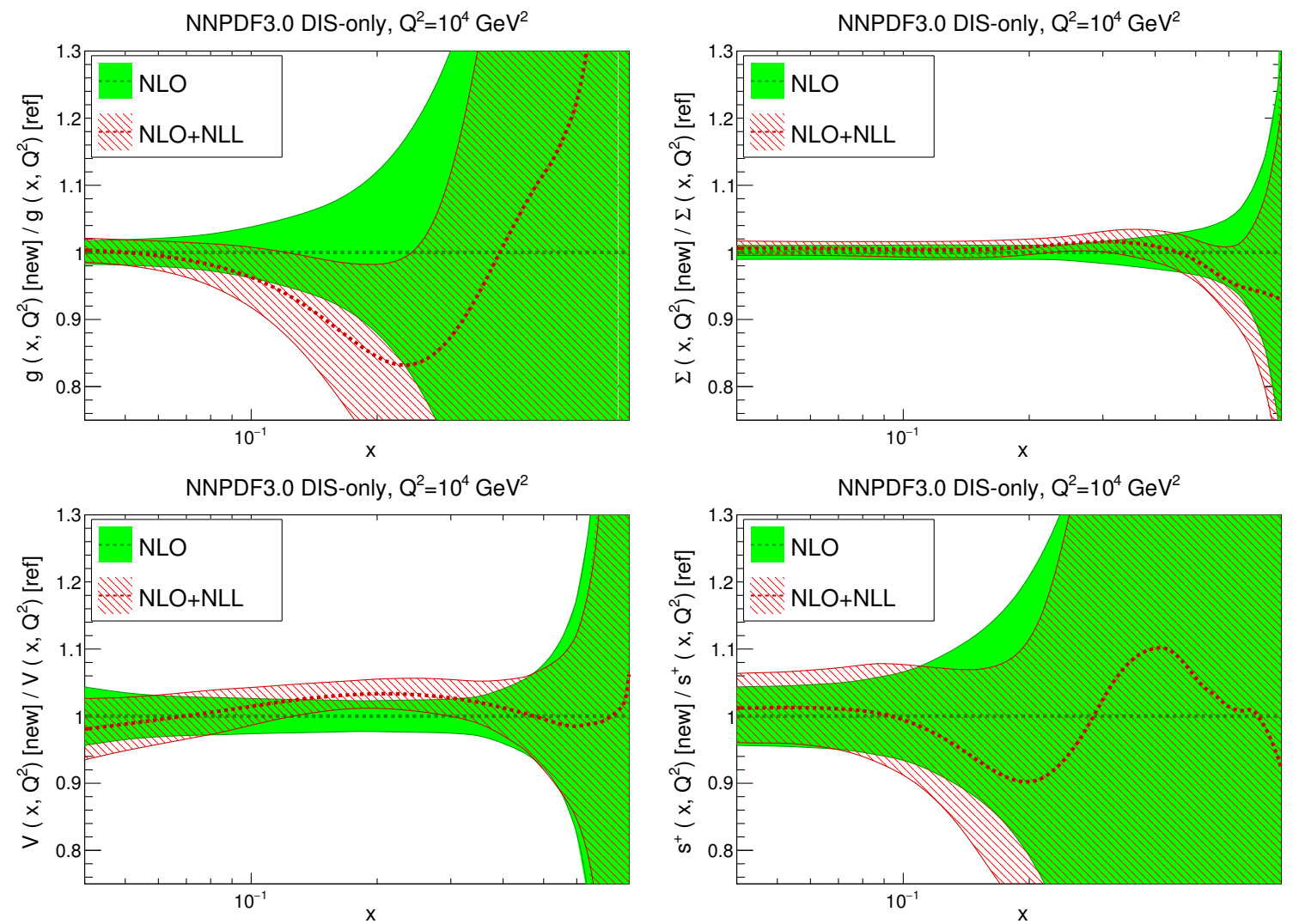

Figure 8. Comparison between the NNPDF3.0 DIS-only NLO fit and the corresponding NLO+NLL fit, for $\alpha_{s}\left(m_{Z}^{2}\right)=0.118$, at a typical LHC scale of $Q^{2}=10^{4} \mathrm{GeV}^{2}$. PDFs are normalised to the central value of the fixed-order fit.

The total $\chi^{2}$ is slightly improved when going from the NNLO to the NNLO+NNLL fit, while it is essentially unaffected in the NLO+NLL case.

Now we turn to study the impact of the resummation on the PDFs themselves. In figure 8 (for the NLO) and in figure 9 (for the NNLO) we compare the NNPDF3.0 DIS-only $(\mathrm{N}) \mathrm{NLO}$ set with $\alpha_{s}\left(m_{Z}^{2}\right)=0.118$, with the corresponding $(\mathrm{N}) \mathrm{NLO}+(\mathrm{N}) \mathrm{NLL}$ threshold resummed PDFs, respectively.

First of all, we note that as expected the inclusion of threshold resummation affects only PDFs at large $x$, for $x \geq 0.1$, which is consistent with the modifications that resummation induces on the DIS structure functions. We also see that the impact on the PDFs is more important at NLL than at NNLL, again as expected since NLL captures part of the NNLO corrections to the DIS structure functions. For the quark PDFs, the effect of resummation is a suppression of the central values for quite large $x$. For example, for the NLO+NLL fit, the total quark singlet $\Sigma\left(x, Q^{2}\right)$ is suppressed by $\sim 5 \%$ at $x \sim 0.6$. One also observes a small enhancement of the valence PDF for $x \sim 0.2$, presumably due to a compensation for the suppression at very large $x$ through the valence sum rules. Therefore, we expect resummation to have phenomenological impact for the calculation of quark-initiated heavy production processes in BSM scenarios, which probe rather large values of $x$. The gluon 

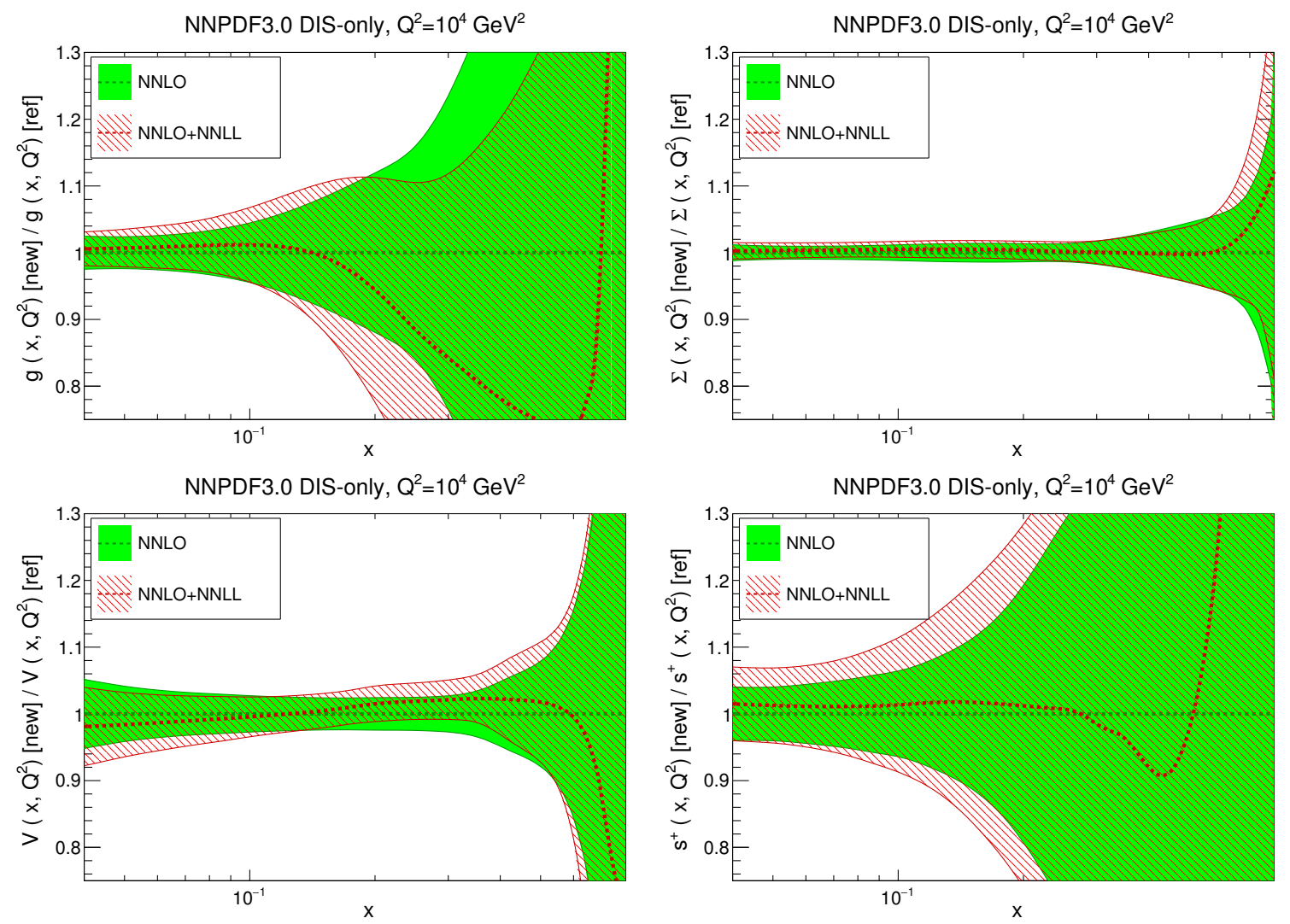

Figure 9. Same as figure 8 now comparing the NNLO DIS-only fit with the corresponding NNLO+NNLL fit.

distribution is also suppressed at large $x$, though PDF uncertainties are too large in a DIS-only fit to make this suppression significant.

\subsection{DIS+DY+top resummed PDFs}

Now we turn our discussion to the impact of threshold resummation in the case of the fits based on the DIS+DY+top dataset. In table 5 we provide the $\chi^{2}$, again using the experimental definition, for all experiments included in the DIS+DY+top threshold resummed fits, at NLO+NLL and NNLO+NNLL, compared with their fixed-order counterparts.

As we can see from table 5 , in the NLO+NLL fits the fit quality of most of the experiments is improved as compared to the fixed-order NLO fit. This is especially marked in the case of SLAC, as in the DIS-only fit, but also for the CHORUS neutrino structure functions, the CDF $Z$ rapidity distribution, ATLAS high-mass DY, LHCb $Z$ rapidity and the top quark pair production. The only exception is the fixed target Drell-Yan data, where resummation makes the $\chi^{2}$ worse. Note however that in the resummed fit the overall balance between experiments in the global fit is modified compared to the fixed-order fit, so this does not necessarily imply that resummation degrades the internal fit quality for 


\begin{tabular}{|c|c|c|c|c|}
\hline Experiment & \multicolumn{4}{|c|}{ NNPDF3.0 DIS+DY+top } \\
\hline NMC & NLO & NNLO & NLO+NLL & NNLO+NNLL \\
\hline SLAC & 1.39 & 1.34 & 1.36 & 1.30 \\
BCDMS & 1.17 & 0.91 & 1.02 & 0.92 \\
CHORUS & 1.20 & 1.25 & 1.23 & 1.28 \\
NuTeV & 1.13 & 1.11 & 1.10 & 1.09 \\
\hline HERA-I & 0.52 & 0.52 & 0.54 & 0.44 \\
ZEUS HERA-II & 1.05 & 1.06 & 1.06 & 1.06 \\
H1 HERA-II & 1.70 & 1.46 & 1.45 & 1.48 \\
HERA charm & 1.26 & 1.28 & 1.70 & 1.78 \\
\hline DY E866 & 1.08 & 1.39 & 1.68 & 1.28 \\
\hline DY E605 & 0.92 & 1.14 & 1.12 & 1.68 \\
\hline CDF $Z$ rap & 1.21 & 1.38 & 1.10 & 1.33 \\
\hline D0 $Z$ rap & 0.57 & 0.62 & 0.67 & 0.66 \\
\hline ATLAS $Z$ 2010 & 0.98 & 1.21 & 1.02 & 1.28 \\
ATLAS high-mass DY & 1.85 & 1.27 & 1.59 & 1.21 \\
\hline CMS 2D DY 2011 & 1.22 & 1.39 & 1.22 & 1.41 \\
\hline LHCb $Z$ rapidity & 0.83 & 1.30 & 0.51 & 1.25 \\
\hline ATLAS CMS top prod & 1.23 & 0.55 & 0.61 & 0.40 \\
\hline Total & 1.233 & 1.264 & 1.246 & 1.269 \\
\hline
\end{tabular}

Table 5. Same as table 4 for the DIS+DY+top fits.

this particular observable. ${ }^{5}$ At the level of total $\chi^{2}$ we see that fixed-order and resummed fits lead to essentially the same value, since in the resummed case the improvement in some experiments is compensated by the deterioration of others.

Turning to the NNLO+NNLL fit results in table 5, we see that now the effect of resummation is more moderate. Effects are small, and also in this case resummation deteriorates the fit quality for the fixed-target Drell-Yan data. Interestingly, the $\chi^{2}$ for the LHCb $Z$ rapidity data, which, being in the forward region, probe rather large values of $x$, improves substantially with the inclusion of resummation, even at NNLL. Given the small differences at the $\chi^{2}$ level, we also expect smaller differences at the PDF level, as in the case of the DIS-only NNLO+NNLL fit.

The comparison of the PDFs between the NLO and NLO+NLL DIS+DY+top fits is shown in figure 10, and the corresponding comparison between the NNLO and NNLO+NNLL fits is found in figure 11. These can be compared with the corresponding DIS-only fits, see figure 8 and figure 9. In the case of the NLO+NLL fit, the trend is similar to that of the DIS-only fit: softer quarks at very large $x$, and a corresponding enhancement

\footnotetext{
${ }^{5}$ We have checked that in a fit based only on HERA data and fixed-target Drell-Yan data, in both the NLO+NLL and NNLO+NNLL fits we get $\chi^{2} \sim 1$ for the Drell-Yan data. Therefore, the deterioration of the $\chi^{2}$ of E866 in the resummed fits can be attributed to tension with other datasets, rather than a failure of the resummation to correctly describe this dataset.
} 

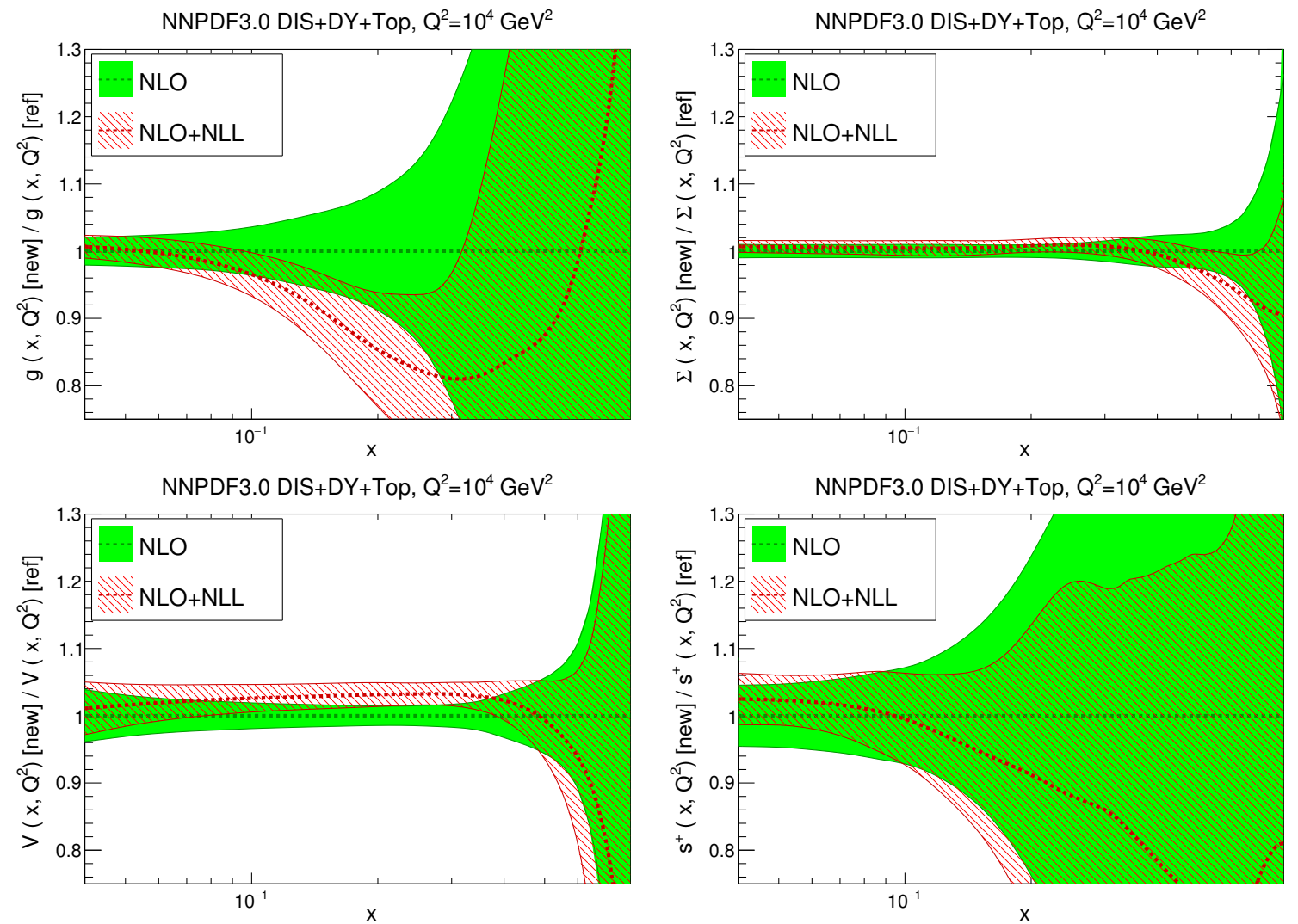

Figure 10. Comparison between the NNPDF3.0 NLO DIS+DY+top fit and the corresponding $\mathrm{NLO}+\mathrm{NLL}$ fit, for $\alpha_{s}\left(m_{Z}^{2}\right)=0.118$, at a typical LHC scale of $Q^{2}=10^{4} \mathrm{GeV}^{2}$.

of the valence distribution at medium $x$. At small $x$, the effect of resummation is negligible as expected. We note that both for the total quark singlet and for the total valence PDF the effect of the resummation has become more significant than in the DIS-only fit, due to the reduction of PDF uncertainties. Indeed, for $\Sigma\left(x, Q^{2}\right)$ for example, the shift of the central value is about $\sim 5 \%$ at $x \simeq 0.5$, and the uncertainty bands of the two fits, although still overlapping, are clearly departing from each other.

At the NNLO+NNLL level, figure 11, the impact of the resummation is as expected even smaller. In particular, the PDF uncertainty bands of the fixed order and resummed fits are quite similar and they overlap in the entire $x$ range. Large shifts in the central values only occur in regions where the PDF uncertainties are large. For example, for the large- $x$ gluon, at $x \sim 0.3$ the resummed central value is $\sim 15 \%$ smaller than the fixed order one, however the PDF uncertainty in this region is substantially larger than the centralvalue shift. At very large $x$, a similar trend can be seen for the total valence PDF, which however exhibits an enhancement at $x \sim 0.3$, which is as big as the PDF uncertainty.

\subsection{Partonic luminosities}

We now study the impact of the inclusion of threshold resummation on PDFs at the level of partonic luminosities. This comparison is useful because it provides direct information on 

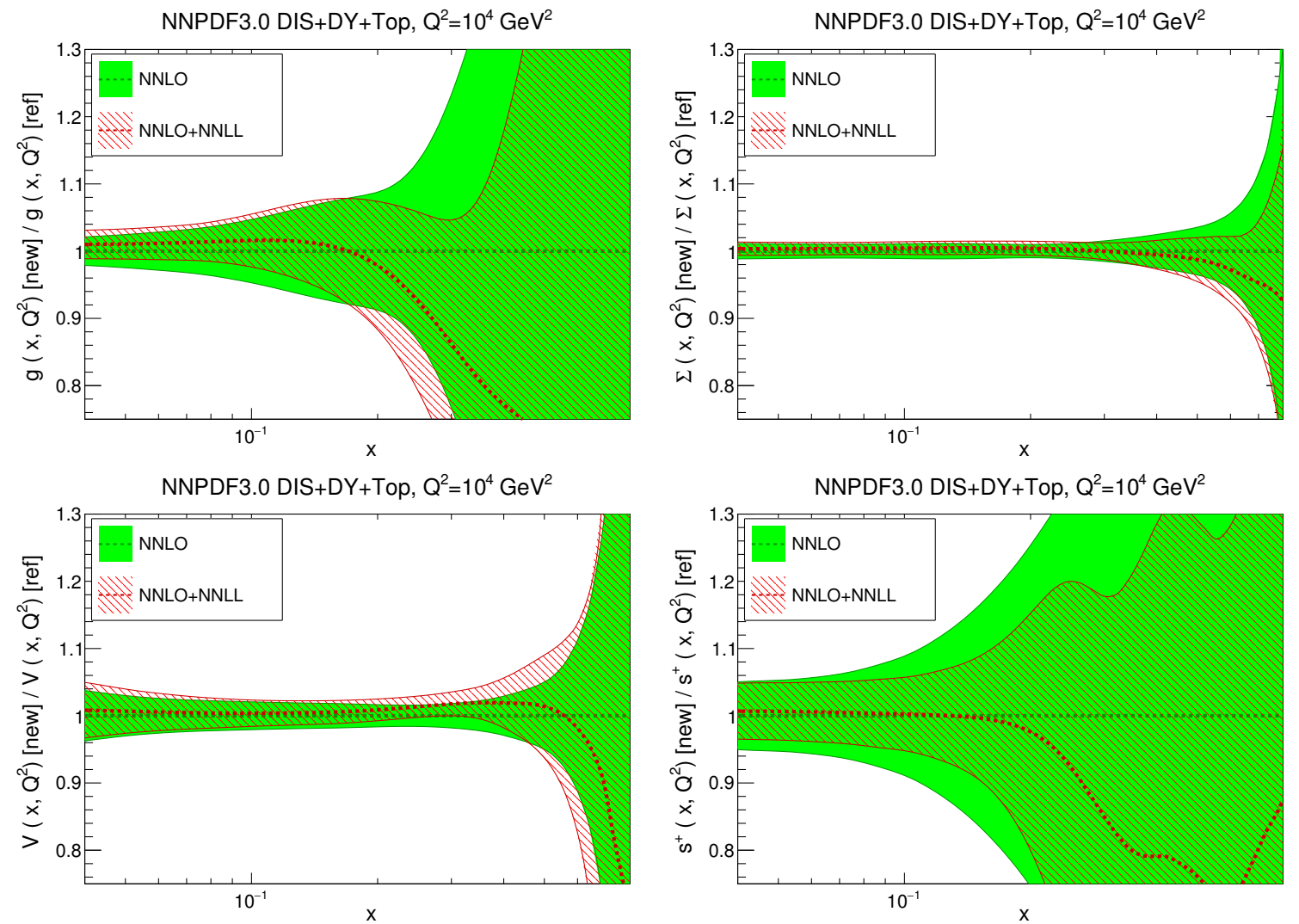

Figure 11. Same as figure 10, now comparing the NNLO DIS+DY+top fit with the corresponding NNLO+NNLL fit.

how the cross sections for the production of a given final state with invariant mass $M_{X}$ will be affected by the inclusion of resummation in the PDFs. It should be emphasised however that in a consistent calculation the impact of the resummation in the PDFs may be compensated by a similar sized effect of the resummation in the partonic matrix elements of the process under consideration. The consistent comparison of LHC cross sections with resummation included both at the PDFs and in the matrix elements is performed in the next section.

We begin by estimating the effect on the PDF luminosities of the reduced dataset used in our baseline fits, as compared to the NNPDF3.0 global fit. Thus in figure 12 we compare the NNPDF3.0 NLO partonic luminosities for $\alpha_{s}\left(m_{Z}^{2}\right)=0.118$, in the global fit and in the DIS+DY+top baseline fit. In the upper plots we show the quark-antiquark and quarkquark luminosities, and in the lower plots the gluon-gluon and gluon-quark luminosities. The calculation has been performed for the LHC $13 \mathrm{TeV}$, as a function of the mass of the final state $M_{X}$, and results are normalised to the central value of the global fit.

As we can see in figure 12, there are some important differences between the global and DIS+DY+top fits. For the $q q$ luminosity, the impact of varying the dataset is small, both in terms of central values and of PDF uncertainties, except at very large values of $M_{X}$. For the $q \bar{q}$ luminosity, the differences are again only sizeable at large $M_{X}$, where the central value of the DIS+DY+top fit is softer than that of the global fit, for instance by $10 \%$ at 

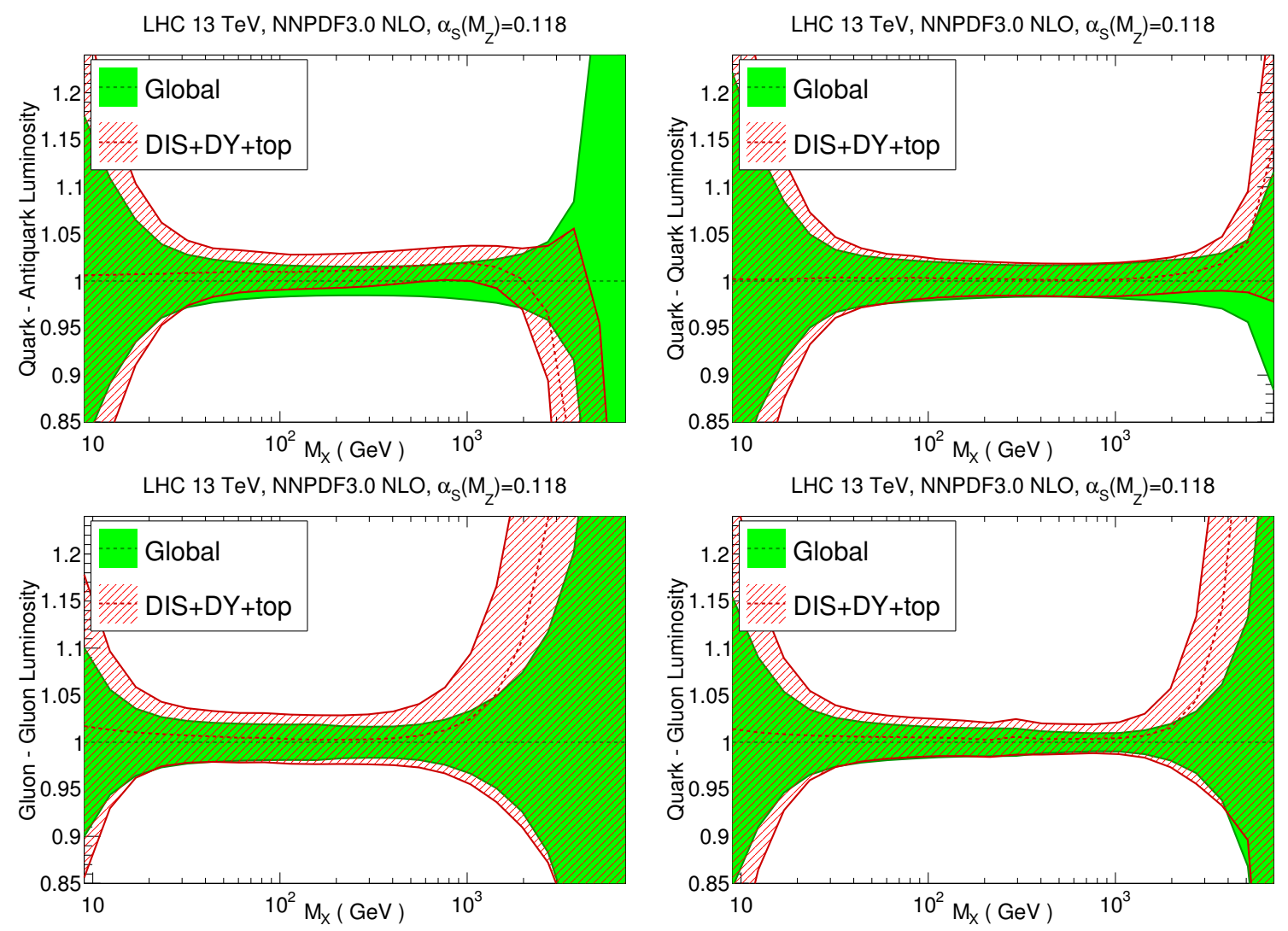

Figure 12. Comparison of the NNPDF3.0 NLO partonic luminosities for $\alpha_{s}\left(m_{Z}^{2}\right)=0.118$, in the global fit and in the DIS+DY+top fit which is used as fixed-order baseline for the resummed fits. In the upper plots we show the quark-antiquark and quark-quark luminosities, and in the lower plots the gluon-gluon and gluon quark luminosities. The calculation has been performed for the LHC $13 \mathrm{TeV}$, as a function of the mass of the final state $M_{X}$, and results are normalised to the central value of the global fit.

$M_{X} \simeq 3 \mathrm{TeV} . \mathrm{PDF}$ uncertainties are similar in the two cases, and the two fits agree within one-sigma. The missing jet data have a stronger impact on the $g g$ and $q g$ luminosities. For instance for the $g g$ luminosity above $0.5 \mathrm{TeV}$, PDF uncertainties increase by a factor two or more. Therefore, in order to consistently assess the impact of the resummation, one should compare the resummed and fixed-order DIS+DY+top fits, rather than the NNPDF3.0 global fit, with the resummed fits presented here.

The comparisons between the DIS+DY+top fixed-order and resummed fits are displayed in figures 13 (at NLL) and 14 (at NNLL). We see that in all cases the fixed-order and resummed fits agree at the level of one sigma, and that the effect of resummation is as expected smaller at NNLL than at NLL. In the comparison between NLO and NLO+NLL, the $q q$ and $q \bar{q}$ luminosities are enhanced by about one sigma for $M_{X} \lesssim 1 \mathrm{TeV}$, while they are suppressed at larger values of $M_{X}$. This behaviour follows from the corresponding PDF comparisons, where quarks are slightly enhanced at $x \simeq 0.1-0.4$ but suppressed for larger values of $x$. This suppression can be sizeable: for $M_{X} \simeq 3 \mathrm{TeV}$ the $q \bar{q}$ luminosity in the NLO+NLL fit is reduced by $\sim 15 \%$. In the $q q$ channel, this suppression instead is small 

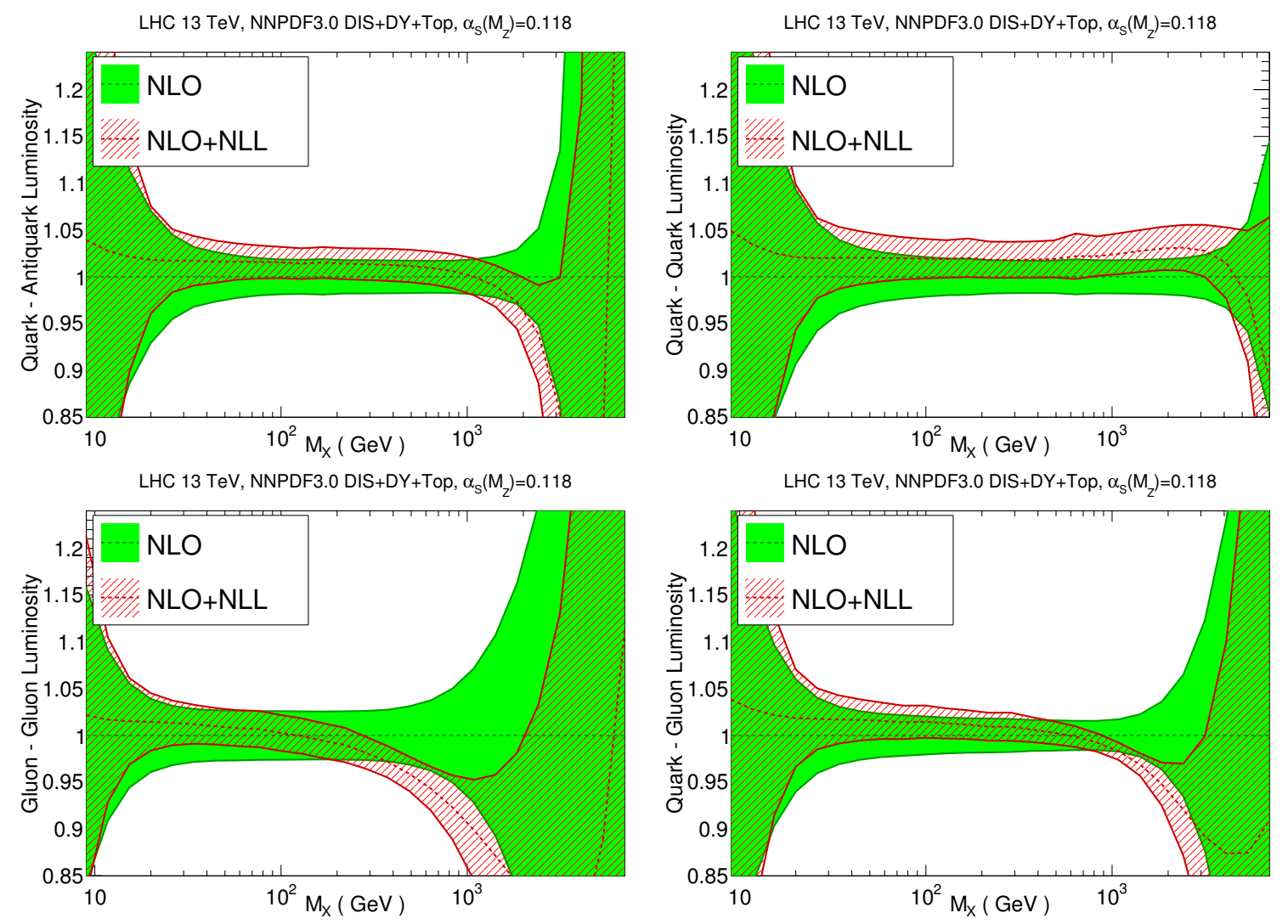

Figure 13. Same as figure 12, now comparing the results of DIS+DY+top fits using either NLO or NLO+NLL calculations.

unless very large values of $M_{X}$ are probed. The $g g$ and $g q$ luminosities are also suppressed at large invariant masses, for instance for $g g$ the suppression is already $\sim 10 \%$ at $1 \mathrm{TeV}$, though still consistent with the fixed-order fit within the large PDF uncertainties.

From the corresponding comparison between the NNLO and NNLO+NNLL fits, shown in figure 14, we see that the effects of resummation are very small everywhere except for the largest values of $M_{X}$. The central values of the $q \bar{q}, g g$ and $q g$ luminosities exhibit some suppression at very large $M_{X}$, but this suppression is not relevant when compared to the PDF uncertainties. From the comparison in figure 14 we thus conclude that the impact of threshold resummation in a global PDF analysis is only relevant at NLO, while at NNLO it appears to be negligible, at least with the current PDF uncertainties. If future data leads to substantial reduction of PDF uncertainties at large- $x$, threshold resummation could be relevant even for NNLO fits.

\section{Resummed PDFs: implications for LHC phenomenology}

In this section we discuss the implications of the NLO+NLL and NNLO+NNLL resummed PDF sets for LHC phenomenology. Our aim is to quantify, for a variety of processes, the difference between using consistently NLO+NLL and NNLO+NNLL calculations at the 

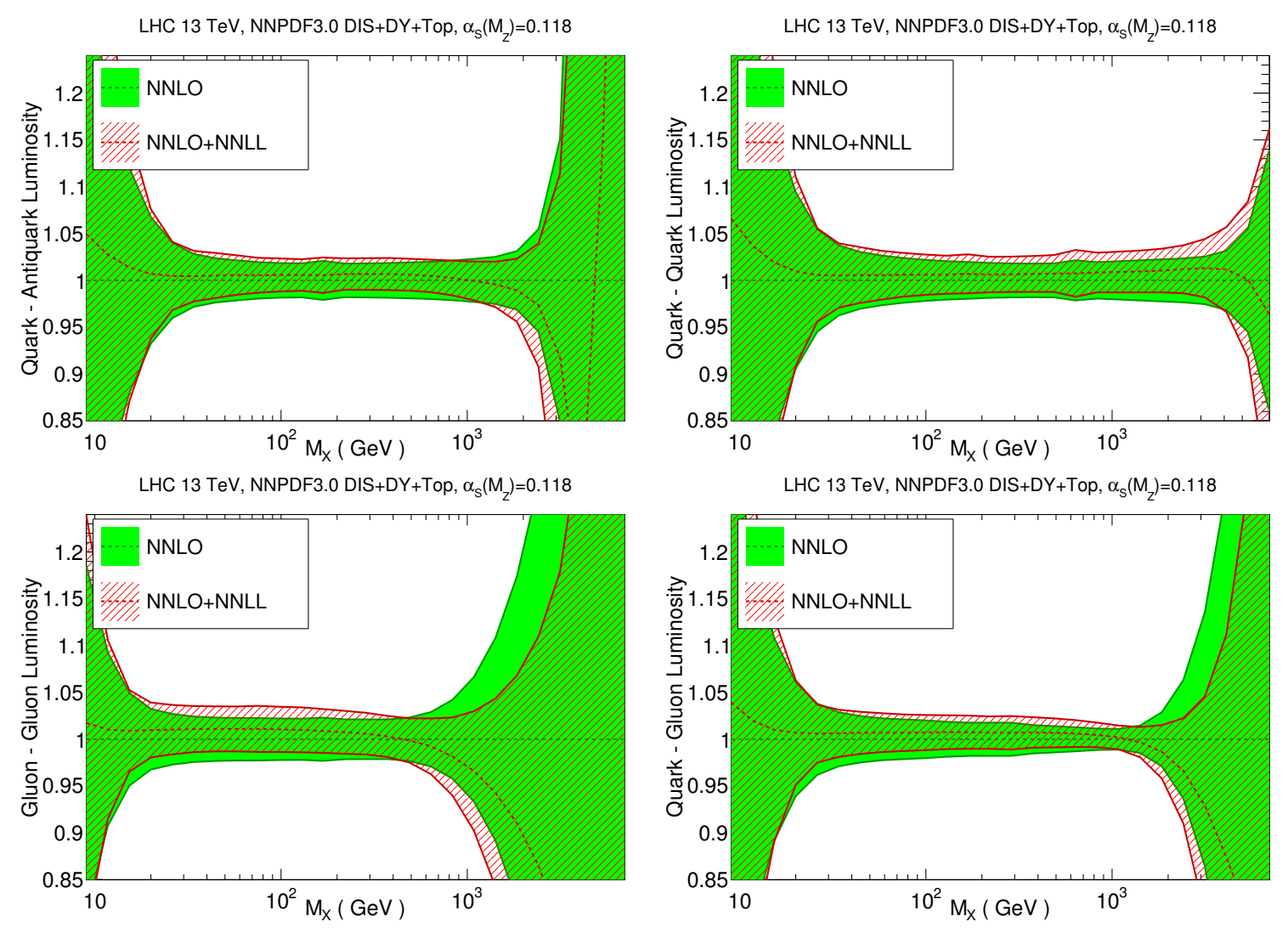

Figure 14. Same as figure 13, this time comparing the NNLO and NNLO+NNLL fits.

level of both PDFs and matrix elements, and the usual (but inconsistent) approach of using resummed partonic cross sections with fixed-order PDFs.

For illustration, we consider three representative LHC processes for which resummed calculations are publicly available, either at the level of total cross sections or of differential invariant-mass distributions. We start by considering Higgs production in gluon fusion, both for $m_{H}=125 \mathrm{GeV}$ and for a heavy BSM Higgs-like neutral scalar. Note that the current recommendation of the Higgs Cross section Working group for inclusive Higgs production in gluon fusion is based on the NNLO+NNLL calculation $[35,173]$. We then consider threshold resummation for the invariant mass distributions of dileptons in the highmass Drell-Yan process, which is important in many New Physics searches, for example for $Z^{\prime}$ searches. Finally we study the invariant mass distribution of supersymmetric lepton (slepton) pair production, a typical final state analysed in electroweak SUSY searches. While Higgs production is driven by the $g g$ luminosity, both high-mass Drell-Yan and slepton pair production are driven by the quark-antiquark luminosity, which is reasonably well constrained even with the reduced dataset used in the present fits.

A variety of other interesting processes are available in which resummed PDFs should be relevant, including top quark differential distributions [62, 63, 174], squark and gluino pair production $[65,67]$ or stop quark pair production [69]. However for most of these processes the corresponding resummation codes are not publicly available. 

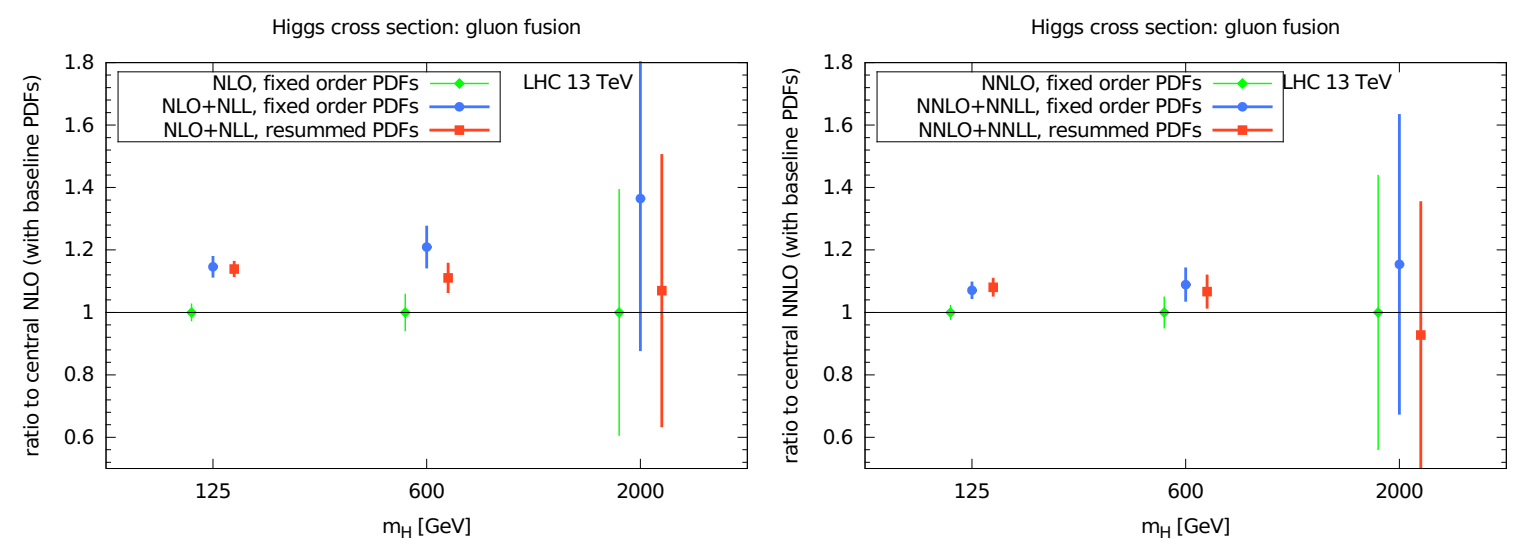

Figure 15. Left: the total cross section for Higgs production in gluon fusion at the LHC $13 \mathrm{TeV}$ for different values of the Higgs mass, comparing the predictions of NLO fixed-order with that of NLO+NLL resummed calculations (using either fixed order or resummed PDFs). Results are shown normalised to the central prediction of the fixed order NLO calculation. Right: the same comparison now performed at NNLO. The calculation has been performed using the ggHiggs code.

\subsection{SM and BSM Higgs production in gluon fusion}

The accurate calculation of Higgs production via gluon fusion is an essential component of the LHC program, since it is required in order to extract Higgs couplings from the ATLAS and CMS measurements. As a result of the recent calculation of the inclusive cross section at $\mathrm{N}^{3} \mathrm{LO}$ [24], PDF uncertainties are now one of the dominant theory uncertainties. In addition to the characterisation of the SM Higgs boson, many New Physics scenarios predict heavy Higgs-like bosons [175-177], and thus it is also important to provide accurate predictions for heavy Higgs production for these BSM searches.

Using the ggHiggs code [121], in figure 15 we show the predictions for the total cross section for Higgs production in gluon fusion at the LHC $13 \mathrm{TeV}$, comparing the (N)NLO fixed-order results with those of the $(\mathrm{N}) \mathrm{NLO}+(\mathrm{N}) \mathrm{NLL}$ resummed calculations, using either fixed-order or resummed PDFs. The calculation has been performed in the $m_{\text {top }} \rightarrow \infty$ limit and neglecting finite-width effects, which is sufficient for current purposes. All results are normalised to the central value of the fixed-order $(\mathrm{N}) \mathrm{NLO}$ calculation, and we provide three different values of the Higgs mass: $m_{H}=125 \mathrm{GeV}, 600 \mathrm{GeV}$ and $2 \mathrm{TeV}$.

The comparisons in figure 15 are interesting because they show that for the production of heavy final states that probe large values of $x$ in the gluon PDF, including resummation in the PDFs can cancel out the effect of the resummation in the matrix element. In the case of the NLO calculation, the SM Higgs cross section is not affected by resummation of the PDFs, but already for $m_{H}=600 \mathrm{GeV}$, the inclusion of resummed PDFs cancels almost half of the enhancement in the hadronic cross section that arises from resummation of the matrix element. For an even heavier Higgs, with $m_{H}=2 \mathrm{TeV}$, the consistent NLO+NLL calculation is essentially identical to the NLO result. The trend is similar at NNLO, though of course in this case the effect of perturbative corrections beyond the fixed-order NNLO 

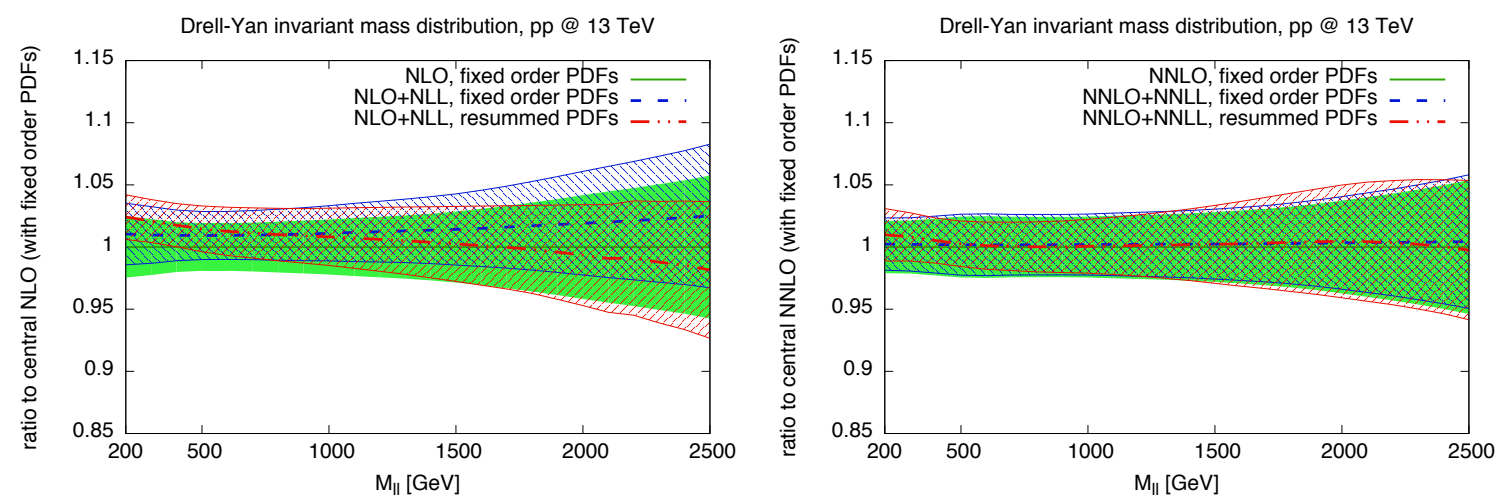

Figure 16. Left: dilepton invariant mass distribution for high-mass neutral current Drell-Yan production at the LHC $13 \mathrm{TeV}$, comparing the predictions of fixed-order with that of resummed calculations. Results are shown normalised to the central prediction of the fixed-order NLO calculation. Right: the same comparison at NNLO.

calculation is smaller. Note also that PDF uncertainties are substantial at large Higgs masses, partly because of the lack of jet data in the baseline and resummed fits.

Our results demonstrate that using consistently resummed PDFs for SM Higgs production at the LHC has no effect, and therefore puts on a more solid ground the current HXSWG recommendation, which is based on fixed-order PDFs. This observation is in agreement with the findings of ref. [178] regarding the (lack of) need of $\mathrm{N}^{3} \mathrm{LO}$ PDFs for the SM Higgs production cross section at $\mathrm{N}^{3} \mathrm{LO}$.

\subsection{High-Mass Drell-Yan dilepton mass distributions}

At the LHC, high-mass Drell-Yan is one of the most important processes when looking for new physics, in particular for new electroweak sectors. For instance, ATLAS and CMS have explored a number of BSM signatures in the high-mass tail of neutral-current Drell-Yan production [179-181], such as $Z^{\prime}$ bosons which appear in several new physics scenarios. It is therefore interesting to assess the effect of including consistently threshold resummation both in the PDFs and in matrix elements, compared to including it only in the matrix element while using fixed-order PDFs.

In figure 16 we show the dilepton invariant mass distribution for high-mass neutral current Drell-Yan production at the LHC $13 \mathrm{TeV}$, comparing the predictions of fixed-order and resummed calculations. The fixed-order NLO and NNLO predictions have been computed with the code Vrap supplemented with threshold resummation as provided by TROLL. In figure 16 we show the predictions for the dilepton invariant mass distribution, comparing the $(\mathrm{N}) \mathrm{NLO}$ fixed-order results with those of the $(\mathrm{N}) \mathrm{NLO}+(\mathrm{N}) \mathrm{NLL}$ resummed calculations, using either fixed-order or resummed PDFs. The latter comparison quantifies the mismatch when resummed calculations are used with fixed-order PDFs.

The results are qualitatively consistent with those of the Higgs cross sections in figure 15. First, we see that even at NLO and at large invariant masses the effect of threshold resummation is moderate: the NLL correction amounts (for fixed-order NLO PDF) to 
about $4 \%$ at $M_{l l}=2.5 \mathrm{TeV}$, which is within the current PDF uncertainty. Including the effect of the resummation in the PDFs consistently cancels this effect, for example in the range for $M_{l l} \in[1.5,2.5] \mathrm{TeV}$ the central value of the NLO+NLL calculation agrees with the fixed-order NLO result by less than one percent. At NNLO the impact of resummation is completely negligible, both at the level of the PDFs and of the matrix elements.

\subsection{Supersymmetric particle production}

The theoretical predictions for high-mass supersymmetric pair production at hadron colliders are currently made at NLO, supplemented with either NLL or NNLL resummation of threshold logarithms. In particular, the NLO+NLL resummed calculations of refs. $[182,183]$ have been used to produce the benchmark production cross sections at $\sqrt{s}=7 \mathrm{TeV}$ and $13 \mathrm{TeV}$ that are used as by ATLAS and CMS in the theoretical interpretation of their searches for supersymmetry [184-187].

An important limitation of these predictions is the mismatch between the fixed-order PDFs and the resummed partonic cross sections, which should be more important at highmasses, precisely the crucial region for New Physics searches. Thanks to the availability, for the first time, of general-purpose resummed PDFs, it is now possible to consistently combine resummed PDFs and matrix elements into a single calculation. It is beyond the scope of this work to present a comprehensive study of the impact of NLO+NLL PDFs for generic supersymmetric processes. However, for illustrative purposes, in this section we will use the public code Resummino [70-72] to compare the effect of resummed PDFs in the context of NLO+NLL predictions for electroweak supersymmetric particle pair production at the LHC, in particular for slepton pair production.

Resummino computes resummed and matched predictions for supersymmetric particle production at hadron colliders up to the NLO+NLL level. Currently the processes implemented include gaugino-pair production and slepton-pair production. These final states are characteristic signatures in electroweak SUSY searches at the LHC [188-191]. Resummino is able to compute total cross sections as well as invariant-mass and transverse-momentum distributions. In this study we focus on the invariant-mass distribution for slepton pair production. Note that the production of sleptons (like many other electroweak SUSY processes) is mostly sensitive to the $q \bar{q}$ luminosity; other processes, such as squark and gluino pair production, would be sensitive to other PDF combinations such as $q g$ and $g g$.

In figure 17 we show the results of the NLO+NLL calculation of the invariant mass distribution for slepton pair production at the LHC $13 \mathrm{TeV}$ obtained with Resummino, using both the NLO and NLO+NLL NNPDF3.0 DIS+DY+top PDFs as input. Results are shown as ratios with respect to the NLO calculation, using consistently the NLO baseline PDFs. The settings of the SUSY calculation are the default ones in Resummino. We use a slepton mass of $m_{\tilde{l}}=564 \mathrm{GeV}$.

The comparison displayed in figure 17 is interesting for a variety of reasons. First of all, we see that, using the NLO PDFs as input, the NLO+NLL calculation (i.e. with resummation included only in the matrix element) enhances the cross section by several percent, from $2 \%$ at $M_{\tilde{l l}} \sim 1.2 \mathrm{TeV}$ up to $5 \%$ at $M_{\tilde{l l}} \sim 3 \mathrm{TeV}$. On the other hand, in the consistent calculation in which resummation is included both at the level of PDFs and of 
Slepton pair invariant mass, pp @ $13 \mathrm{TeV}, \mathrm{m}_{\mathrm{l}}=564 \mathrm{GeV}$.

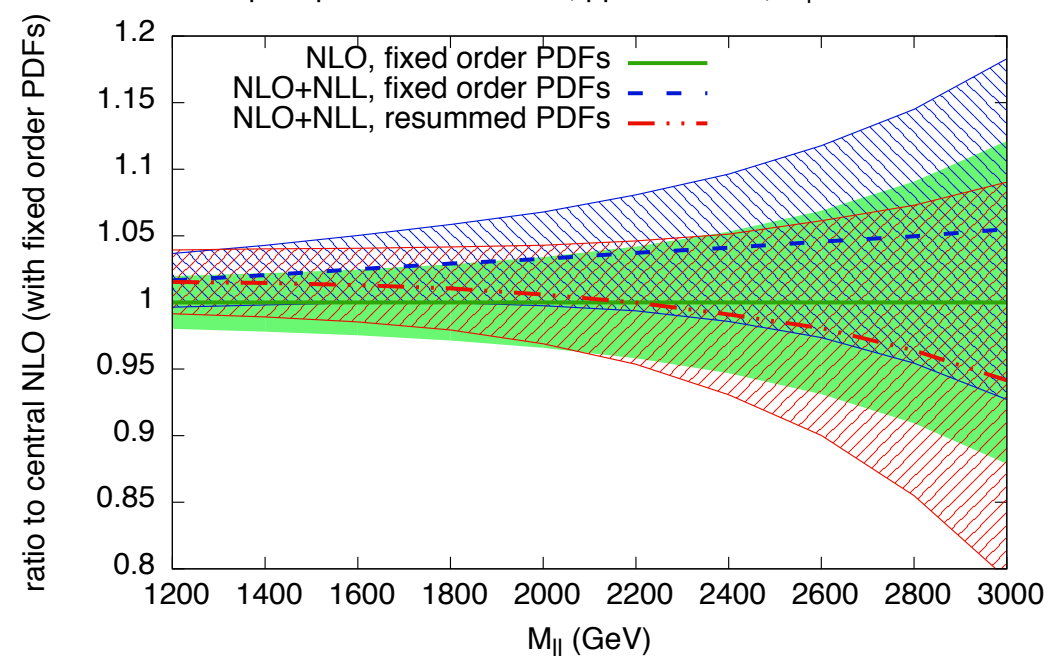

Figure 17. The NLO+NLL calculation of the invariant mass distribution for slepton pair production at the LHC $13 \mathrm{TeV}$ using the Resummino program, using both the NLO and NLO+NLL NNPDF3.0 DIS+DY+top PDFs as input. Results are shown as ratios with respect to the NLO calculation, using consistently the NLO baseline PDFs. The settings of the SUSY calculation are the default ones in Resummino. We use a slepton mass of $m_{\tilde{l}}=564 \mathrm{GeV}$.

matrix elements, this increase is only seen around $M_{\tilde{l}} \sim 1.2 \mathrm{TeV}$. For higher invariant masses up to $M_{\tilde{l l}} \sim 2.5 \mathrm{TeV}$ or so the effect of resummation in the PDFs cancels the one originating from the matrix elements, and the consistent NLO+NLL calculation is essentially the same as the NLO one. For even higher masses, the NLO+NLL calculation is suppressed compared to the NLO calculation by up to $5 \%$ at $M_{\tilde{l}} \sim 3 \mathrm{TeV}$, though in this region PDF uncertainties are very large.

The results of figure 17 are consistent with the behavior of the $q \bar{q}$ luminosity shown in figure 13. In particular, for an invariant mass of $M \simeq 1 \mathrm{TeV}$, the NLO and NLO+NLL PDF luminosities are essentially the same, while for $M \simeq 3 \mathrm{TeV}$ the NLO+NLL luminosity is suppressed by a factor of approximately $10 \%$, a similar amount as that inferred from the Resummino plot of figure 17. This illustrates that, for those processes which are dominated by a single partonic luminosity, one can approximately correct a NLO+NLL matrix element calculation using the ratio of PDF luminosities. This is also consistent with the high-mass Drell-Yan results of figure 16, which are also driven by the $q \bar{q}$ luminosity.

In summary, even though we have been able to explore only a limited number of resummed calculations for LHC processes, a consistent trend appears. When the produced final state has an invariant mass far from threshold, the use of resummed PDFs has a rather small effect. However, for heavy final states, the main effect of the resummation of the PDFs is to compensate the effect of the resummation in the matrix element, so that the consistent $(\mathrm{N}) \mathrm{NLO}+(\mathrm{N}) \mathrm{NLL}$ calculation is rather closer to the fixed-order (N)NLO result. This shows that using resummation only in the matrix element but not in the PDF can be 
misleading, since it may overestimate cross sections and invariant mass distributions. This is particularly the case for NLO+NLL calculations, because at NNLO+NNLL the effect of the resummation is much smaller, since much of it has already been accounted for in the NNLO fixed-order corrections.

In conclusion, one should in general always use resummed PDFs with threshold resummed matrix elements. This said, even if the central value of the consistent (N)NLO+ $(\mathrm{N})$ NLL calculation is reasonably close to the original fixed-order (N)NLO result, it is in general still better to use the resummed calculation, since these benefit for instance from reduced scale dependence, and thus smaller theoretical uncertainties.

\section{Summary}

In this paper we have presented for the first time global fits of parton distributions extracted at NLO+NLL and NNLO+NNLL accuracy, where the fixed-order partonic cross sections have been systematically improved using soft-gluon threshold resummation. We find that the main effect of threshold resummation is to suppress the PDFs in the large- $x$ region, as expected given that the fit compensates from the resummation-induced increment in the partonic cross sections used in the PDF fit. This suppression is important for all PDF flavors for $x \gtrsim 0.1$, while at intermediate values of $x, 0.01 \lesssim x \lesssim 0.1$, the quark PDFs are instead somewhat enhanced due to the sum rules. For smaller values of $x, x \lesssim 0.01$, the effect of resummation becomes completely negligible.

At the level of PDF luminosities at the LHC $13 \mathrm{TeV}$, we find that at the NLO+NLL level the suppression induced by resummation in the PDFs starts to become important for $M_{X} \gtrsim 400 \mathrm{GeV}$ in the $g g$ channel, $M_{X} \gtrsim 1 \mathrm{TeV}$ in the $q \bar{q}$ and $q g$ channels, and $M_{X} \gtrsim$ $5 \mathrm{TeV}$ for the $q q$ channel. The trend is similar at NNLO, but in this case differences between fixed-order and resummed PDFs are much smaller. We also find that fixed-order and resummed PDFs differ by at most one sigma throughout all the range of $M_{X}$.

We have investigated the corresponding implications at the level of resummed LHC cross sections for three different processes: SM and BSM Higgs production in gluon fusion, high-mass Drell-Yan pair production and slepton pair production. We find that the effect of consistently including resummation in the PDFs can compensate the enhancement from resummation of the partonic cross sections, if $M_{X}$ is large enough. For the production of final states with lower $M_{X}$, the effect of PDF resummation is negligible. This trend is likely to be general: when fitting to data, PDFs adjust to absorb the effect of the resummation in the partonic cross sections, and this compensation inevitably persists when extrapolating to predictions for new processes.

Our results illustrate the importance of using the same perturbative order in all the components that enter hadronic cross sections: the use of fixed-order PDFs with resummed matrix elements can lead to misleading results, especially at high invariant masses, a region crucial for new physics searches. The partial cancellation between resummation in PDFs and in matrix elements indicates that consistent resummed calculations can be closer to fixed-order results. This said, even in the case of a complete cancellation, use of resummation would still be advantageous, because of the reduced scale uncertainty. For these 
reasons, we expect that the resummed NNPDF3.0 sets will provide a crucial ingredient, missing so far, to improve the precision of all-order resummed calculations for the LHC, and in particular those used in searches for new physics.

The main limitation of the present work is that, since resummed calculations are not available for all processes included in the NNPDF3.0 global fit, we have restricted the dataset in the resummed fits (and the corresponding baseline) to those processes that can be consistently resummed. In particular, we have had to exclude the inclusive jet production data and $W$ lepton rapidity distributions. Hence, our resummed sets generate larger PDF uncertainties than the NNPDF3.0 global PDFs, especially for gluon-initiated processes. It is thus important in the future to provide resummed calculations for these missing processes, in order to produce a truly global resummed PDF analysis.

With the recent start-up of the LHC Run II, the need for precision calculations is even more pressing than in Run I, since precision could be the key to uncovering new physics. The results of this paper offer for the first time fully consistent threshold resummed calculations, which constitute state-of-the-art accuracy for a number of important LHC processes, from Higgs to supersymmetric particle production. Therefore, the resummed NNPDF3.0 sets presented here achieve a new milestone in the program of precision phenomenology at the LHC.

Delivery. The resummed calculations for DIS structure function and Drell-Yan distributions used in this work have been obtained with the new code TROLL, version v3.0. This code is publicly available from

$$
\text { http://www.ge.infn.it/ bonvini/troll/ }
$$

and can be used by any interested parties to compute their own predictions for resummed observables.

Our resummed sets are available in the LHAPDF6 format from the authors upon request. The available sets are:

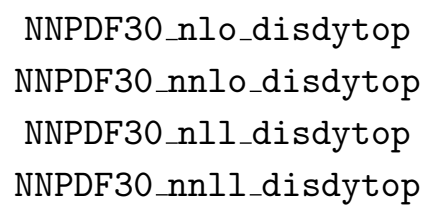

which stand for the NLO and NNLO baseline fits, and their NLO+NLL and NNLO+NNLL resummed counterparts. All these PDF sets are provided for $\alpha_{s}\left(m_{Z}^{2}\right)=0.118$ with a maximum of $n_{f}=5$ active flavors.

\section{Acknowledgments}

We thank all the members of the NNPDF Collaboration, and especially S. Forte and G. Ridolfi, for discussions and encouragement during this project. We thank B. Pecjak and A. Kulesza for discussions. S.M. and M.U. would like to thank the Rudolf Peierls Centre for Theoretical Physics, at Oxford University for hospitality during the course of 
this work. The work of S.M. is supported by the U.S. National Science Foundation, under grant PHY-0969510, the LHC Theory Initiative. J.R. is supported by an STFC Rutherford Fellowship ST/K005227/1. M.B., J.R. and L.R. are supported by an European Research Council Starting Grant "PDF4BSM". S.C. is supported in part by an Italian PRIN2010 grant and by a European Investment Bank EIBURS grant. V.B. is supported by the ERC grant 291377, LHCtheory: Theoretical predictions and analyses of LHC physics: advancing the precision frontier.

Open Access. This article is distributed under the terms of the Creative Commons Attribution License (CC-BY 4.0), which permits any use, distribution and reproduction in any medium, provided the original author(s) and source are credited.

\section{References}

[1] S. Forte and G. Watt, Progress in the determination of the partonic structure of the proton, Ann. Rev. Nucl. Part. Sci. 63 (2013) 291 [arXiv:1301.6754] [INSPIRE].

[2] R.D. Ball et al., Parton distribution benchmarking with LHC data, JHEP 04 (2013) 125 [arXiv: 1211.5142] [INSPIRE].

[3] E. Perez and E. Rizvi, The quark and gluon structure of the proton, Rept. Prog. Phys. 76 (2013) 046201 [arXiv:1208.1178] [INSPIRE].

[4] J. Rojo et al., The PDF 4 LHC report on PDFs and LHC data: results from Run I and preparation for Run II, arXiv: 1507.00556 [INSPIRE].

[5] G. Watt, Parton distribution function dependence of benchmark standard model total cross sections at the 7 TeV LHC, JHEP 09 (2011) 069 [arXiv:1106.5788] [INSPIRE].

[6] J.R. Andersen et al., Les Houches 2013: Physics at TeV colliders. Standard model working group report, arXiv:1405.1067 [INSPIRE].

[7] M. Czakon, P. Fiedler and A. Mitov, Total top-quark pair-production cross section at hadron colliders through $O\left(\alpha_{S}^{4}\right)$, Phys. Rev. Lett. 110 (2013) 252004 [arXiv:1303.6254] [InSPIRE].

[8] S. Catani, L. Cieri, D. de Florian, G. Ferrera and M. Grazzini, Diphoton production at hadron colliders: a fully-differential QCD calculation at $N N L O$, Phys. Rev. Lett. 108 (2012) 072001 [arXiv:1110.2375] [InSPIRE].

[9] J. Currie, A. Gehrmann-De Ridder, E.W.N. Glover and J. Pires, NNLO QCD corrections to jet production at hadron colliders from gluon scattering, JHEP 01 (2014) 110 [arXiv: 1310.3993] [INSPIRE].

[10] X. Chen, T. Gehrmann, E.W.N. Glover and M. Jaquier, Precise QCD predictions for the production of Higgs + jet final states, Phys. Lett. B 740 (2015) 147 [arXiv:1408.5325] [INSPIRE].

[11] R. Boughezal, F. Caola, K. Melnikov, F. Petriello and M. Schulze, Higgs boson production in association with a jet at next-to-next-to-leading order, Phys. Rev. Lett. 115 (2015) 082003 [arXiv: 1504.07922] [INSPIRE].

[12] R. Boughezal, F. Caola, K. Melnikov, F. Petriello and M. Schulze, Higgs boson production in association with a jet at next-to-next-to-leading order in perturbative $Q C D$, JHEP 06 (2013) 072 [arXiv:1302.6216] [INSPIRE]. 
[13] M. Brucherseifer, F. Caola and K. Melnikov, On the NNLO QCD corrections to single-top production at the LHC, Phys. Lett. B 736 (2014) 58 [arXiv:1404.7116] [InSPIRE].

[14] D. de Florian and J. Mazzitelli, Higgs boson pair production at next-to-next-to-leading order in QCD, Phys. Rev. Lett. 111 (2013) 201801 [arXiv:1309.6594] [INSPIRE].

[15] P. Bolzoni, F. Maltoni, S.-O. Moch and M. Zaro, Higgs production via vector-boson fusion at NNLO in QCD, Phys. Rev. Lett. 105 (2010) 011801 [arXiv:1003.4451] [INSPIRE].

[16] M. Cacciari, F.A. Dreyer, A. Karlberg, G.P. Salam and G. Zanderighi, Fully differential vector-boson-fusion Higgs production at next-to-next-to-leading order, Phys. Rev. Lett. 115 (2015) 082002 [arXiv:1506.02660] [INSPIRE].

[17] M. Grazzini, S. Kallweit and D. Rathlev, $W \gamma$ and $Z \gamma$ production at the LHC in NNLO QCD, JHEP 07 (2015) 085 [arXiv: 1504.01330] [INSPIRE].

[18] T. Gehrmann et al., $W^{+} W^{-}$production at hadron colliders in next to next to leading order QCD, Phys. Rev. Lett. 113 (2014) 212001 [arXiv:1408.5243] [INSPIRE].

[19] G. Ferrera, M. Grazzini and F. Tramontano, Associated ZH production at hadron colliders: the fully differential NNLO QCD calculation, Phys. Lett. B 740 (2015) 51 [arXiv: 1407.4747] [INSPIRE].

[20] F. Cascioli et al., ZZ production at hadron colliders in NNLO QCD, Phys. Lett. B 735 (2014) 311 [arXiv:1405.2219] [INSPIRE].

[21] M. Grazzini, S. Kallweit, D. Rathlev and A. Torre, $Z \gamma$ production at hadron colliders in NNLO QCD, Phys. Lett. B 731 (2014) 204 [arXiv:1309.7000] [INSPIRE].

[22] R. Boughezal, C. Focke, X. Liu and F. Petriello, $W$-boson production in association with a jet at next-to-next-to-leading order in perturbative $Q C D$,

Phys. Rev. Lett. 115 (2015) 062002 [arXiv:1504.02131] [INSPIRE].

[23] R. Boughezal, C. Focke, W. Giele, X. Liu and F. Petriello, Higgs boson production in association with a jet at NNLO using jettiness subtraction, Phys. Lett. B 748 (2015) 5 [arXiv: 1505. 03893] [INSPIRE].

[24] C. Anastasiou, C. Duhr, F. Dulat, F. Herzog and B. Mistlberger, Higgs boson gluon-fusion production in QCD at three loops, Phys. Rev. Lett. 114 (2015) 212001 [arXiv:1503.06056] [INSPIRE].

[25] M. Dittmar et al., Working Group I: Parton distributions. Summary report for the HERA-LHC Workshop Proceedings, hep-ph/0511119 [INSPIRE].

[26] G. Luisoni and S. Marzani, QCD resummation for hadronic final states, J. Phys. G 42 (2015) 103101 [arXiv: 1505.04084] [InSPIRE].

[27] M. Ciafaloni, D. Colferai, G.P. Salam and A.M. Stasto, A matrix formulation for small-x singlet evolution, JHEP 08 (2007) 046 [arXiv:0707.1453] [INSPIRE].

[28] R.D. Ball, Resummation of hadroproduction cross-sections at high energy, Nucl. Phys. B 796 (2008) 137 [arXiv:0708.1277] [INSPIRE].

[29] G. Altarelli, R.D. Ball and S. Forte, Small x resummation with quarks: deep-inelastic scattering, Nucl. Phys. B 799 (2008) 199 [arXiv:0802.0032] [INSPIRE].

[30] S. Marzani and R.D. Ball, High energy resummation of Drell-Yan processes, Nucl. Phys. B 814 (2009) 246 [arXiv:0812.3602] [INSPIRE]. 
[31] F. Caola, S. Forte and J. Rojo, Deviations from NLO QCD evolution in inclusive HERA data, Phys. Lett. B 686 (2010) 127 [arXiv:0910.3143] [InSPIRE].

[32] F. Caola, S. Forte and J. Rojo, HERA data and DGLAP evolution: theory and phenomenology, Nucl. Phys. A 854 (2011) 32 [arXiv:1007.5405] [INSPIRE].

[33] ZEUS and H1 collaborations, H. Abramowicz et al., Combination of measurements of inclusive deep inelastic $e^{ \pm} p$ scattering cross sections and QCD analysis of HERA data, arXiv: 1506.06042 [INSPIRE].

[34] S. Catani, D. de Florian, M. Grazzini and P. Nason, Soft gluon resummation for Higgs boson production at hadron colliders, JHEP 07 (2003) 028 [hep-ph/0306211] [INSPIRE].

[35] D. de Florian and M. Grazzini, Higgs production through gluon fusion: updated cross sections at the Tevatron and the LHC, Phys. Lett. B 674 (2009) 291 [arXiv:0901.2427] [INSPIRE].

[36] M. Bonvini and S. Marzani, Resummed Higgs cross section at $N^{3} L L$, JHEP 09 (2014) 007 [arXiv: 1405.3654] [INSPIRE].

[37] M. Bonvini and L. Rottoli, Three loop soft function for $N^{3} L L^{\prime}$ gluon fusion Higgs production in soft-collinear effective theory, Phys. Rev. D 91 (2015) 051301 [arXiv: 1412.3791] [INSPIRE].

[38] S. Catani, L. Cieri, D. de Florian, G. Ferrera and M. Grazzini, Threshold resummation at $N^{3} L L$ accuracy and soft-virtual cross sections at $N^{3} L O$, Nucl. Phys. B 888 (2014) 75 [arXiv: 1405.4827] [INSPIRE].

[39] R.V. Harlander, A. Kulesza, V. Theeuwes and T. Zirke, Soft gluon resummation for gluon-induced Higgs Strahlung, JHEP 11 (2014) 082 [arXiv:1410.0217] [INSPIRE].

[40] D. de Florian and J. Mazzitelli, Higgs pair production at next-to-next-to-leading logarithmic accuracy at the LHC, JHEP 09 (2015) 053 [arXiv: 1505.07122] [INSPIRE].

[41] G.F. Sterman, Summation of large corrections to short distance hadronic cross-sections, Nucl. Phys. B 281 (1987) 310 [InSPIRE].

[42] S. Catani and L. Trentadue, Resummation of the QCD perturbative series for hard processes, Nucl. Phys. B 327 (1989) 323 [InSPIRE].

[43] S. Catani and L. Trentadue, Comment on QCD exponentiation at large $x$, Nucl. Phys. B 353 (1991) 183 [INSPIRE].

[44] A. Vogt, Next-to-next-to-leading logarithmic threshold resummation for deep inelastic scattering and the Drell-Yan process, Phys. Lett. B 497 (2001) 228 [hep-ph/0010146] [INSPIRE].

[45] S. Moch, J.A.M. Vermaseren and A. Vogt, Higher-order corrections in threshold resummation, Nucl. Phys. B 726 (2005) 317 [hep-ph/0506288] [INSPIRE].

[46] T. Becher, M. Neubert and B.D. Pecjak, Factorization and momentum-space resummation in deep-inelastic scattering, JHEP 01 (2007) 076 [hep-ph/0607228] [INSPIRE].

[47] A.V. Manohar, Deep inelastic scattering as $x \rightarrow 1$ using soft collinear effective theory, Phys. Rev. D 68 (2003) 114019 [hep-ph/0309176] [INSPIRE].

[48] A. Idilbi, X.-d. Ji and F. Yuan, Resummation of threshold logarithms in effective field theory for DIS, Drell-Yan and Higgs production, Nucl. Phys. B 753 (2006) 42 [hep-ph/0605068] [INSPIRE]. 
[49] S. Moch and A. Vogt, Higher-order soft corrections to lepton pair and Higgs boson production, Phys. Lett. B 631 (2005) 48 [hep-ph/0508265] [INSPIRE].

[50] E. Laenen and L. Magnea, Threshold resummation for electroweak annihilation from DIS data, Phys. Lett. B 632 (2006) 270 [hep-ph/0508284] [INSPIRE].

[51] T. Ahmed, M. Mahakhud, N. Rana and V. Ravindran, Drell-Yan production at threshold to third order in QCD, Phys. Rev. Lett. 113 (2014) 112002 [arXiv:1404.0366] [INSPIRE].

[52] E. Laenen and G.F. Sterman, Resummation for Drell-Yan differential distributions, FERMILAB-CONF-92-359-T, ITP-SB-92-69 (1992) [INSPIRE].

[53] P. Bolzoni, Threshold resummation of Drell-Yan rapidity distributions, Phys. Lett. B 643 (2006) 325 [hep-ph/0609073] [INSPIRE].

[54] A. Mukherjee and W. Vogelsang, Threshold resummation for W-boson production at RHIC, Phys. Rev. D 73 (2006) 074005 [hep-ph/0601162] [INSPIRE].

[55] V. Ravindran, J. Smith and W.L. van Neerven, QCD threshold corrections to di-lepton and Higgs rapidity distributions beyond $N^{2}$ LO, Nucl. Phys. B 767 (2007) 100 [hep-ph/0608308] [INSPIRE].

[56] V. Ravindran and J. Smith, Threshold corrections to rapidity distributions of $Z$ and $W^{ \pm}$ bosons beyond $N^{2} L O$ at hadron colliders, Phys. Rev. D 76 (2007) 114004 [arXiv:0708.1689] [INSPIRE].

[57] T. Becher, M. Neubert and G. Xu, Dynamical threshold enhancement and resummation in Drell-Yan production, JHEP 07 (2008) 030 [arXiv:0710.0680] [INSPIRE].

[58] M. Bonvini, S. Forte and G. Ridolfi, Soft gluon resummation of Drell-Yan rapidity distributions: theory and phenomenology, Nucl. Phys. B 847 (2011) 93 [arXiv:1009.5691] [INSPIRE].

[59] M. Czakon, A. Mitov and G.F. Sterman, Threshold resummation for top-pair hadroproduction to next-to-next-to-leading log, Phys. Rev. D 80 (2009) 074017 [arXiv:0907.1790] [INSPIRE].

[60] M. Beneke, P. Falgari, S. Klein and C. Schwinn, Hadronic top-quark pair production with NNLL threshold resummation, Nucl. Phys. B 855 (2012) 695 [arXiv:1109.1536] [InSPIRE].

[61] N. Kidonakis, E. Laenen, S. Moch and R. Vogt, Sudakov resummation and finite order expansions of heavy quark hadroproduction cross-sections, Phys. Rev. D 64 (2001) 114001 [hep-ph/0105041] [INSPIRE].

[62] V. Ahrens, A. Ferroglia, M. Neubert, B.D. Pecjak and L.-L. Yang, RG-improved

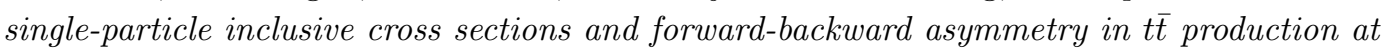
hadron colliders, JHEP 09 (2011) 070 [arXiv:1103.0550] [INSPIRE].

[63] A. Ferroglia, S. Marzani, B.D. Pecjak and L.L. Yang, Boosted top production: factorization and resummation for single-particle inclusive distributions, JHEP 01 (2014) 028 [arXiv:1310.3836] [INSPIRE].

[64] W. Beenakker et al., Squark and gluino hadroproduction, Int. J. Mod. Phys. A 26 (2011) 2637 [arXiv:1105.1110] [INSPIRE].

[65] W. Beenakker et al., NNLL resummation for squark-antisquark pair production at the LHC, JHEP 01 (2012) 076 [arXiv:1110.2446] [InSPIRE]. 
[66] P. Falgari, C. Schwinn and C. Wever, NLL soft and Coulomb resummation for squark and gluino production at the LHC, JHEP 06 (2012) 052 [arXiv: 1202.2260] [INSPIRE].

[67] W. Beenakker et al., NNLL resummation for squark and gluino production at the LHC, JHEP 12 (2014) 023 [arXiv: 1404.3134] [INSPIRE].

[68] W. Beenakker et al., Supersymmetric top and bottom squark production at hadron colliders, JHEP 08 (2010) 098 [arXiv: 1006.4771] [INSPIRE].

[69] A. Broggio, A. Ferroglia, M. Neubert, L. Vernazza and L.L. Yang, NNLL momentum-space resummation for stop-pair production at the LHC, JHEP 03 (2014) 066 [arXiv:1312.4540] [INSPIRE].

[70] B. Fuks, M. Klasen, D.R. Lamprea and M. Rothering, Precision predictions for electroweak superpartner production at hadron colliders with Resummino,

Eur. Phys. J. C 73 (2013) 2480 [arXiv:1304.0790] [InSPIRE].

[71] B. Fuks, M. Klasen, D.R. Lamprea and M. Rothering, Revisiting slepton pair production at the Large Hadron Collider, JHEP 01 (2014) 168 [arXiv:1310.2621] [INSPIRE].

[72] G. Bozzi, B. Fuks and M. Klasen, Threshold resummation for slepton-pair production at hadron colliders, Nucl. Phys. B 777 (2007) 157 [hep-ph/0701202] [INSPIRE].

[73] A. Broggio, M. Neubert and L. Vernazza, Soft-gluon resummation for slepton-pair production at hadron colliders, JHEP 05 (2012) 151 [arXiv:1111.6624] [INSPIRE].

[74] G.P. Korchemsky, Asymptotics of the Altarelli-Parisi-Lipatov evolution kernels of parton distributions, Mod. Phys. Lett. A 4 (1989) 1257 [INSPIRE].

[75] S. Albino and R.D. Ball, Soft resummation of quark anomalous dimensions and coefficient functions in $\overline{\mathrm{MS}}$ factorization, Phys. Lett. B 513 (2001) 93 [hep-ph/0011133] [INSPIRE].

[76] G. Curci, W. Furmanski and R. Petronzio, Evolution of parton densities beyond leading order: the nonsinglet case, Nucl. Phys. B 175 (1980) 27 [INSPIRE].

[77] A. Gonzalez-Arroyo and C. Lopez, Second order contributions to the structure functions in deep inelastic scattering (III). The singlet case, Nucl. Phys. B 166 (1980) 429 [INSPIRE].

[78] E.G. Floratos, D.A. Ross and C.T. Sachrajda, Higher-order effects in asymptotically free gauge theories: the anomalous dimensions of Wilson operators, Nucl. Phys. B 129 (1977) 66 [Erratum ibid. B 139 (1978) 545] [InSPIRE].

[79] E.G. Floratos, D.A. Ross and C.T. Sachrajda, Higher-order effects in asymptotically free gauge theories: (II). Flavor singlet Wilson operators and coefficient functions,

Nucl. Phys. B 152 (1979) 493 [inSPIRE].

[80] S. Moch, J.A.M. Vermaseren and A. Vogt, The three-loop splitting functions in QCD: the non-singlet case, Nucl. Phys. B 688 (2004) 101 [hep-ph/0403192] [INSPIRE].

[81] A. Vogt, S. Moch and J.A.M. Vermaseren, The three-loop splitting functions in QCD: the singlet case, Nucl. Phys. B 691 (2004) 129 [hep-ph/0404111] [INSPIRE].

[82] D. Westmark, Threshold resummation and the determinations of parton distribution functions, arXiv:1309.7989 [INSPIRE].

[83] G. Corcella and L. Magnea, Soft-gluon resummation effects on parton distributions, Phys. Rev. D 72 (2005) 074017 [hep-ph/0506278] [inSPIRE].

[84] N. Sato, Threshold resummation in direct photon production and its implications on the large-x gluon PDF, arXiv:1309.7995 [INSPIRE]. 
[85] A. Accardi, D.P. Anderle and F. Ringer, Interplay of threshold resummation and hadron mass corrections in deep inelastic processes, Phys. Rev. D 91 (2015) 034008 [arXiv:1411.3649] [INSPIRE].

[86] NNPDF collaboration, L. Del Debbio, S. Forte, J.I. Latorre, A. Piccione and J. Rojo, Unbiased determination of the proton structure function $F_{2}^{p}$ with faithful uncertainty estimation, JHEP 03 (2005) 080 [hep-ph/0501067] [INSPIRE].

[87] NNPDF collaboration, L. Del Debbio, S. Forte, J.I. Latorre, A. Piccione and J. Rojo, Neural network determination of parton distributions: the nonsinglet case, JHEP 03 (2007) 039 [hep-ph/0701127] [INSPIRE].

[88] NNPDF collaboration, R.D. Ball et al., A determination of parton distributions with faithful uncertainty estimation, Nucl. Phys. B 809 (2009) 1

[Erratum ibid. B 816 (2009) 293] [arXiv: 0808.1231] [INSPIRE].

[89] NNPDF collaboration, R.D. Ball et al., Precision determination of electroweak parameters and the strange content of the proton from neutrino deep-inelastic scattering, Nucl. Phys. B 823 (2009) 195 [arXiv:0906.1958] [INSPIRE].

[90] R.D. Ball et al., A first unbiased global NLO determination of parton distributions and their uncertainties, Nucl. Phys. B 838 (2010) 136 [arXiv:1002.4407] [INSPIRE].

[91] R.D. Ball et al., Impact of heavy quark masses on parton distributions and LHC phenomenology, Nucl. Phys. B 849 (2011) 296 [arXiv:1101.1300] [INSPIRE].

[92] NNPDF collaboration, R.D. Ball et al., Unbiased global determination of parton distributions and their uncertainties at NNLO and at LO, Nucl. Phys. B 855 (2012) 153 [arXiv:1107.2652] [INSPIRE].

[93] R.D. Ball et al., Parton distributions with LHC data, Nucl. Phys. B 867 (2013) 244 [arXiv: 1207.1303] [INSPIRE].

[94] N. Kidonakis and J.F. Owens, Effects of higher-order threshold corrections in high- $E_{T}$ jet production, Phys. Rev. D 63 (2001) 054019 [hep-ph/0007268] [INSPIRE].

[95] D. de Florian, P. Hinderer, A. Mukherjee, F. Ringer and W. Vogelsang, Approximate next-to-next-to-leading order corrections to hadronic jet production, Phys. Rev. Lett. 112 (2014) 082001 [arXiv:1310.7192] [INSPIRE].

[96] S. Carrazza and J. Pires, Perturbative QCD description of jet data from LHC Run-I and Tevatron Run-II, JHEP 10 (2014) 145 [arXiv:1407.7031] [INSPIRE].

[97] NNPDF collaboration, R.D. Ball et al., Parton distributions for the LHC Run II, JHEP 04 (2015) 040 [arXiv:1410.8849] [InSPIRE].

[98] S. Catani, M.L. Mangano and P. Nason, Sudakov resummation for prompt photon production in hadron collisions, JHEP 07 (1998) 024 [hep-ph/9806484] [INSPIRE].

[99] T. Becher, A. Broggio and A. Ferroglia, Introduction to soft-collinear effective theory, arXiv:1410.1892 [INSPIRE].

[100] M. Bonvini, S. Forte, M. Ghezzi and G. Ridolfi, Threshold resummation in SCET vs. perturbative QCD: an analytic comparison, Nucl. Phys. B 861 (2012) 337 [arXiv:1201.6364] [INSPIRE]. 
[101] M. Bonvini, S. Forte, M. Ghezzi and G. Ridolfi, The scale of soft resummation in SCET vs. perturbative QCD, Nucl. Phys. Proc. Suppl. 241-242 (2013) 121 [arXiv:1301.4502] [INSPIRE].

[102] M. Bonvini, S. Forte, G. Ridolfi and L. Rottoli, Resummation prescriptions and ambiguities in SCET vs. direct QCD: Higgs production as a case study, JHEP 01 (2015) 046 [arXiv: 1409.0864] [INSPIRE].

[103] G. Sterman and M. Zeng, Quantifying comparisons of threshold resummations, JHEP 05 (2014) 132 [arXiv: 1312.5397] [INSPIRE].

[104] M. Cacciari, M. Czakon, M. Mangano, A. Mitov and P. Nason, Top-pair production at hadron colliders with next-to-next-to-leading logarithmic soft-gluon resummation, Phys. Lett. B 710 (2012) 612 [arXiv:1111.5869] [inSPIRE].

[105] N. Kidonakis, G. Oderda and G.F. Sterman, Threshold resummation for dijet cross-sections, Nucl. Phys. B 525 (1998) 299 [hep-ph/9801268] [INSPIRE].

[106] D. de Florian and W. Vogelsang, Threshold resummation for the inclusive-hadron cross-section in pp collisions, Phys. Rev. D 71 (2005) 114004 [hep-ph/0501258] [INSPIRE].

[107] M. Dasgupta and G.P. Salam, Resummation of nonglobal QCD observables, Phys. Lett. B 512 (2001) 323 [hep-ph/0104277] [INSPIRE].

[108] A. Banfi and M. Dasgupta, Problems in resumming interjet energy flows with $k_{t}$ clustering, Phys. Lett. B 628 (2005) 49 [hep-ph/0508159] [INSPIRE].

[109] Y. Delenda, R. Appleby, M. Dasgupta and A. Banfi, On QCD resummation with $k_{t}$ clustering, JHEP 12 (2006) 044 [hep-ph/0610242] [INSPIRE].

[110] S. Catani, M. Grazzini and A. Torre, Soft-gluon resummation for single-particle inclusive hadroproduction at high transverse momentum, Nucl. Phys. B 874 (2013) 720 [arXiv: 1305.3870] [INSPIRE].

[111] A. Broggio, A. Ferroglia, B.D. Pecjak and Z. Zhang, NNLO hard functions in massless QCD, JHEP 12 (2014) 005 [arXiv:1409.5294] [INSPIRE].

[112] P. Hinderer, F. Ringer, G.F. Sterman and W. Vogelsang, Toward NNLL threshold resummation for hadron pair production in hadronic collisions, Phys. Rev. D 91 (2015) 014016 [arXiv:1411.3149] [INSPIRE].

[113] R.D. Ball, M. Bonvini, S. Forte, S. Marzani and G. Ridolfi, Higgs production in gluon fusion beyond NNLO, Nucl. Phys. B 874 (2013) 746 [arXiv:1303.3590] [InSPIRE].

[114] M. Bonvini, R.D. Ball, S. Forte, S. Marzani and G. Ridolfi, Updated Higgs cross section at approximate $N^{3} L O, J$. Phys. G 41 (2014) 095002 [arXiv:1404.3204] [INSPIRE].

[115] C. Muselli, M. Bonvini, S. Forte, S. Marzani and G. Ridolfi, Top quark pair production beyond NNLO, JHEP 08 (2015) 076 [arXiv:1505.02006] [INSPIRE].

[116] S. Catani, M.L. Mangano, P. Nason and L. Trentadue, The resummation of soft gluons in hadronic collisions, Nucl. Phys. B 478 (1996) 273 [hep-ph/9604351] [INSPIRE].

[117] S. Forte, G. Ridolfi, J. Rojo and M. Ubiali, Borel resummation of soft gluon radiation and higher twists, Phys. Lett. B 635 (2006) 313 [hep-ph/0601048] [INSPIRE].

[118] R. Abbate, S. Forte and G. Ridolfi, A new prescription for soft gluon resummation, Phys. Lett. B 657 (2007) 55 [arXiv:0707.2452] [INSPIRE]. 
[119] M. Bonvini, S. Forte and G. Ridolfi, Borel resummation of transverse momentum distributions, Nucl. Phys. B 808 (2009) 347 [arXiv:0807.3830] [INSPIRE].

[120] M. Bonvini, Resummation of soft and hard gluon radiation in perturbative QCD, arXiv: 1212.0480 [INSPIRE].

[121] http://www.ge.infn.it/ bonvini/higgs/.

[122] J.A.M. Vermaseren, A. Vogt and S. Moch, The third-order QCD corrections to deep-inelastic scattering by photon exchange, Nucl. Phys. B 724 (2005) 3 [hep-ph/0504242] [INSPIRE].

[123] M. Czakon, M.L. Mangano, A. Mitov and J. Rojo, Constraints on the gluon PDF from top quark pair production at hadron colliders, JHEP 07 (2013) 167 [arXiv:1303.7215] [INSPIRE].

[124] New Muon collaboration, M. Arneodo et al., Accurate measurement of $F_{2}^{d} / F_{2}^{p}$ and $R^{d}-R^{p}$, Nucl. Phys. B 487 (1997) 3 [hep-ex/9611022] [INSPIRE].

[125] New Muon collaboration, M. Arneodo et al., Measurement of the proton and deuteron structure functions, $F_{2}^{p}$ and $F_{2}^{d}$, and of the ratio $\sigma_{L} / \sigma_{T}$, Nucl. Phys. B 483 (1997) 3 [hep-ph/9610231] [INSPIRE].

[126] BCDMS collaboration, A.C. Benvenuti et al., A high statistics measurement of the proton structure functions $F_{2}\left(x, Q^{2}\right)$ and $R$ from deep inelastic muon scattering at high $Q^{2}$, Phys. Lett. B 223 (1989) 485 [INSPIRE].

[127] BCDMS collaboration, A.C. Benvenuti et al., A high statistics measurement of the deuteron structure functions $F_{2}\left(X, Q^{2}\right)$ and $R$ from deep inelastic muon scattering at high $Q^{2}$, Phys. Lett. B 237 (1990) 592 [inSPIRE].

[128] L.W. Whitlow, E.M. Riordan, S. Dasu, S. Rock and A. Bodek, Precise measurements of the proton and deuteron structure functions from a global analysis of the SLAC deep inelastic electron scattering cross-sections, Phys. Lett. B 282 (1992) 475 [INSPIRE].

[129] CHORUS collaboration, G. Onengut et al., Measurement of nucleon structure functions in neutrino scattering, Phys. Lett. B 632 (2006) 65 [INSPIRE].

[130] NuTeV collaboration, M. Goncharov et al., Precise measurement of dimuon production cross-sections in muon neutrino Fe and muon anti-neutrino Fe deep inelastic scattering at the Tevatron, Phys. Rev. D 64 (2001) 112006 [hep-ex/0102049] [INSPIRE].

[131] ZEUS and H1 collaborations, F.D. Aaron et al., Combined measurement and QCD analysis of the inclusive $e^{ \pm} p$ scattering cross sections at HERA, JHEP 01 (2010) 109 [arXiv:0911.0884] [INSPIRE].

[132] ZEUS collaboration, S. Chekanov et al., Measurement of high- $Q^{2}$ neutral current deep inelastic $e^{-} p$ scattering cross sections with a longitudinally polarised electron beam at HERA, Eur. Phys. J. C 62 (2009) 625 [arXiv:0901.2385] [INSPIRE].

[133] ZEUS collaboration, S. Chekanov et al., Measurement of charged current deep inelastic scattering cross sections with a longitudinally polarised electron beam at HERA, Eur. Phys. J. C 61 (2009) 223 [arXiv:0812.4620] [INSPIRE].

[134] ZEUS collaboration, H. Abramowicz et al., Measurement of high- $Q^{2}$ neutral current deep inelastic $e^{+} p$ scattering cross sections with a longitudinally polarized positron beam at HERA, Phys. Rev. D 87 (2013) 052014 [arXiv:1208.6138] [InSPIRE]. 
[135] ZEUS collaboration, H. Abramowicz et al., Measurement of high- $Q^{2}$ charged current deep inelastic scattering cross sections with a longitudinally polarised positron beam at HERA, Eur. Phys. J. C 70 (2010) 945 [arXiv: 1008.3493] [InSPIRE].

[136] H1 collaboration, F.D. Aaron et al., Inclusive deep inelastic scattering at high $Q^{2}$ with longitudinally polarised lepton beams at HERA, JHEP 09 (2012) 061 [arXiv:1206.7007] [INSPIRE].

[137] H1 collaboration, F.D. Aaron et al., Measurement of the inclusive $e^{ \pm} p$ scattering cross section at high inelasticity $y$ and of the structure function $F_{L}$,

Eur. Phys. J. C 71 (2011) 1579 [arXiv:1012.4355] [inSPIRE].

[138] ZEUS and H1 collaborations, H. Abramowicz et al., Combination and QCD analysis of charm production cross section measurements in deep-inelastic ep scattering at HERA, Eur. Phys. J. C 73 (2013) 2311 [arXiv:1211.1182] [inSPIRE].

[139] NuSEa collaboration, R.S. Towell et al., Improved measurement of the $\bar{d} / \bar{u}$ asymmetry in the nucleon sea, Phys. Rev. D 64 (2001) 052002 [hep-ex/0103030] [INSPIRE].

[140] NuSEa collaboration, J.C. Webb et al., Absolute Drell-Yan dimuon cross-sections in $800 \mathrm{GeV} / \mathrm{c} \mathrm{pp}$ and pd collisions, hep-ex/0302019 [INSPIRE].

[141] J.C. Webb, Measurement of continuum dimuon production in $800-\mathrm{GeV} / \mathrm{c}$ proton nucleon collisions, hep-ex/0301031 [INSPIRE].

[142] G. Moreno et al., Dimuon production in proton-copper collisions at $\sqrt{s}=38.8 \mathrm{GeV}$, Phys. Rev. D 43 (1991) 2815 [InSPIRE].

[143] CDF collaboration, T.A. Aaltonen et al., Measurement of $d \sigma / d y$ of Drell-Yan $e^{+} e^{-}$pairs in the $Z$ mass region from p pollisions at $\sqrt{s}=1.96$ TeV, Phys. Lett. B 692 (2010) 232 [arXiv:0908.3914] [INSPIRE].

[144] CDF collaboration, A. Abulencia et al., Measurement of the inclusive jet cross section using the $k_{\mathrm{T}}$ algorithm in $p \bar{p}$ collisions at $\sqrt{s}=1.96 \mathrm{TeV}$ with the CDF II detector, Phys. Rev. D 75 (2007) 092006 [Erratum ibid. D 75 (2007) 119901] [hep-ex/0701051] [INSPIRE].

[145] D0 collaboration, V.M. Abazov et al., Measurement of the shape of the boson rapidity distribution for $p \bar{p} \rightarrow Z / \gamma^{*} \rightarrow e^{+} e^{-}+X$ events produced at $\sqrt{s}$ of $1.96 \mathrm{TeV}$, Phys. Rev. D 76 (2007) 012003 [hep-ex/0702025] [INSPIRE].

[146] ATLAS collaboration, Measurement of the inclusive $W^{ \pm}$and $Z / \gamma^{*}$ cross sections in the $e$ and $\mu$ decay channels in pp collisions at $\sqrt{s}=7 \mathrm{TeV}$ with the ATLAS detector, Phys. Rev. D 85 (2012) 072004 [arXiv: 1109.5141] [INSPIRE].

[147] ATLAS collaboration, Measurement of inclusive jet and dijet production in pp collisions at $\sqrt{s}=7 \mathrm{TeV}$ using the ATLAS detector, Phys. Rev. D 86 (2012) 014022 [arXiv:1112.6297] [INSPIRE].

[148] ATLAS collaboration, Measurement of the inclusive jet cross section in pp collisions at $\sqrt{s}=2.76 \mathrm{TeV}$ and comparison to the inclusive jet cross section at $\sqrt{s}=7 \mathrm{TeV}$ using the ATLAS detector, Eur. Phys. J. C 73 (2013) 2509 [arXiv:1304.4739] [InSPIRE].

[149] ATLAS collaboration, Measurement of the high-mass Drell-Yan differential cross-section in pp collisions at $\sqrt{s}=7 \mathrm{TeV}$ with the ATLAS detector, Phys. Lett. B $7 \mathbf{2 5}$ (2013) 223 [arXiv:1305.4192] [INSPIRE]. 
[150] ATLAS collaboration, Measurement of the transverse momentum distribution of $W$ bosons in pp collisions at $\sqrt{s}=7 \mathrm{TeV}$ with the ATLAS detector, Phys. Rev. D 85 (2012) 012005 [arXiv:1108.6308] [INSPIRE].

[151] CMS collaboration, Measurement of the electron charge asymmetry in inclusive $W$ production in pp collisions at $\sqrt{s}=7$ TeV, Phys. Rev. Lett. 109 (2012) 111806 [arXiv:1206.2598] [INSPIRE].

[152] CMS collaboration, Measurement of the muon charge asymmetry in inclusive $p p \rightarrow W+X$ production at $\sqrt{s}=7 \mathrm{TeV}$ and an improved determination of light parton distribution functions, Phys. Rev. D 90 (2014) 032004 [arXiv:1312.6283] [INSPIRE].

[153] CMS collaboration, Measurements of differential jet cross sections in proton-proton collisions at $\sqrt{s}=7 \mathrm{TeV}$ with the CMS detector, Phys. Rev. D 87 (2013) 112002 [arXiv: 1212.6660] [INSPIRE].

[154] CMS collaboration, Measurement of associated $W+$ charm production in pp collisions at $\sqrt{s}=7 \mathrm{TeV}$, JHEP 02 (2014) 013 [arXiv:1310.1138] [INSPIRE].

[155] CMS collaboration, Measurement of the differential and double-differential Drell-Yan cross sections in proton-proton collisions at $\sqrt{s}=7 \mathrm{TeV}$, JHEP 12 (2013) 030 [arXiv: 1310.7291] [INSPIRE].

[156] LHCb collaboration, Inclusive $W$ and $Z$ production in the forward region at $\sqrt{s}=7 \mathrm{TeV}$, JHEP 06 (2012) 058 [arXiv:1204.1620] [INSPIRE].

[157] LHCb collaboration, Measurement of the cross-section for $Z \rightarrow e^{+} e^{-}$production in pp collisions at $\sqrt{s}=7 \mathrm{TeV}$, JHEP 02 (2013) 106 [arXiv:1212.4620] [INSPIRE].

[158] ATLAS collaboration, Measurement of the cross section for top-quark pair production in $p p$ collisions at $\sqrt{s}=7 \mathrm{TeV}$ with the ATLAS detector using final states with two high-p $p_{T}$ leptons, JHEP 05 (2012) 059 [arXiv:1202.4892] [INSPIRE].

[159] ATLAS collaboration, Measurement of the t$t \bar{t}$ production cross-section in pp collisions at $\sqrt{s}=7 \mathrm{TeV}$ using kinematic information of lepton+jets events, ATLAS-CONF-2011-121 (2011).

[160] ATLAS collaboration, Measurement of the t $\bar{t}$ production cross-section in pp collisions at $\sqrt{s}=8 \mathrm{TeV}$ using e $\mu$ events with b-tagged jets, ATLAS-CONF-2013-097 (2013).

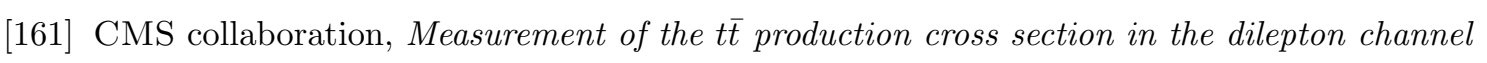
in pp collisions at $\sqrt{s}=8 \mathrm{TeV}$, JHEP 02 (2014) 024 [Erratum ibid. 02 (2014) 102] [arXiv: 1312.7582] [inSPIRE].

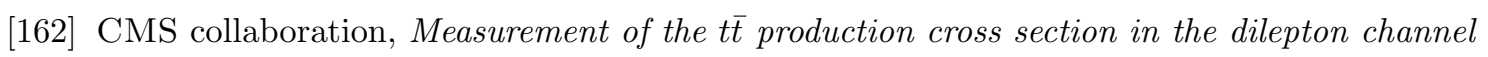
in pp collisions at $\sqrt{s}=7 \mathrm{TeV}$, JHEP 11 (2012) 067 [arXiv:1208.2671] [INSPIRE].

[163] CMS collaboration, Measurement of the t $\bar{t}$ production cross section in pp collisions at $\sqrt{s}=7 \mathrm{TeV}$ with lepton+jets final states, Phys. Lett. B 720 (2013) 83 [arXiv:1212.6682] [INSPIRE].

[164] CLAS collaboration, S. Tkachenko et al., Measurement of the structure function of the nearly free neutron using spectator tagging in inelastic ${ }^{2} H\left(e, e^{\prime} p_{s}\right) X$ scattering with CLAS, Phys. Rev. C 89 (2014) 045206 [arXiv: 1402.2477] [InSPIRE].

[165] S. Forte, E. Laenen, P. Nason and J. Rojo, Heavy quarks in deep-inelastic scattering, Nucl. Phys. B 834 (2010) 116 [arXiv:1001.2312] [INSPIRE]. 
[166] V. Bertone, R. Frederix, S. Frixione, J. Rojo and M. Sutton, aMCfast: automation of fast NLO computations for PDF fits, JHEP 08 (2014) 166 [arXiv: 1406.7693] [INSPIRE].

[167] T. Carli et al., A posteriori inclusion of parton density functions in NLO QCD final-state calculations at hadron colliders: the APPLGRID project, Eur. Phys. J. C 66 (2010) 503 [arXiv:0911.2985] [INSPIRE].

[168] FASTNLO collaboration, M. Wobisch, D. Britzger, T. Kluge, K. Rabbertz and F. Stober, Theory-data comparisons for jet measurements in hadron-induced processes, arXiv:1109.1310 [INSPIRE].

[169] V. Bertone, S. Carrazza and J. Rojo, APFEL: A PDF Evolution Library with QED corrections, Comput. Phys. Commun. 185 (2014) 1647 [arXiv:1310.1394] [INSPIRE].

[170] C. Anastasiou, L.J. Dixon, K. Melnikov and F. Petriello, High precision QCD at hadron colliders: electroweak gauge boson rapidity distributions at NNLO, Phys. Rev. D 69 (2004) 094008 [hep-ph/0312266] [INSPIRE].

[171] M. Beneke et al., Inclusive top-pair production phenomenology with TOPIXS, JHEP 07 (2012) 194 [arXiv: 1206.2454] [INSPIRE].

[172] NNPDF collaboration, R.D. Ball et al., Fitting parton distribution data with multiplicative normalization uncertainties, JHEP 05 (2010) 075 [arXiv:0912.2276] [INSPIRE].

[173] S. Dittmaier et al., Handbook of LHC Higgs cross sections: 2. Differential distributions, arXiv:1201.3084 [INSPIRE].

[174] M. Guzzi, K. Lipka and S.-O. Moch, Top-quark pair production at hadron colliders: differential cross section and phenomenological applications with DiffTop, JHEP 01 (2015) 082 [arXiv:1406.0386] [INSPIRE].

[175] C.-Y. Chen, S. Dawson and M. Sher, Heavy Higgs searches and constraints on two Higgs doublet models, Phys. Rev. D 88 (2013) 015018 [Erratum ibid. D 88 (2013) 039901] [arXiv: 1305.1624] [INSPIRE].

[176] A. Djouadi and J. Quevillon, The MSSM Higgs sector at a high $M_{\mathrm{SUSY}}$ : reopening the low $\tan \beta$ regime and heavy Higgs searches, JHEP 10 (2013) 028 [arXiv:1304.1787] [INSPIRE].

[177] A. Arbey, M. Battaglia and F. Mahmoudi, Supersymmetric heavy Higgs bosons at the LHC, Phys. Rev. D 88 (2013) 015007 [arXiv:1303.7450] [INSPIRE].

[178] S. Forte, A. Isgrò and G. Vita, Do we need $N^{3} L O$ parton distributions?, Phys. Lett. B 731 (2014) 136 [arXiv:1312.6688] [INSPIRE].

[179] ATLAS collaboration, Search for contact interactions and large extra dimensions in the dilepton channel using proton-proton collisions at $\sqrt{s}=8 \mathrm{TeV}$ with the ATLAS detector, Eur. Phys. J. C 74 (2014) 3134 [arXiv:1407.2410] [INSPIRE].

[180] ATLAS collaboration, Search for high-mass dilepton resonances in pp collisions at $\sqrt{s}=8 \mathrm{TeV}$ with the ATLAS detector, Phys. Rev. D 90 (2014) 052005 [arXiv:1405.4123] [INSPIRE].

[181] CMS collaboration, Search for physics beyond the standard model in dilepton mass spectra in proton-proton collisions at $\sqrt{s}=8 \mathrm{TeV}$, JHEP 04 (2015) 025 [arXiv:1412.6302] [INSPIRE].

[182] C. Borschensky et al., Squark and gluino production cross sections in pp collisions at $\sqrt{s}=13,14,33$ and $100 \mathrm{TeV}$, Eur. Phys. J. C 74 (2014) 3174 [arXiv:1407.5066] [INSPIRE]. 
[183] M. Krämer et al., Supersymmetry production cross sections in pp collisions at $\sqrt{s}=7 \mathrm{TeV}$, arXiv: 1206.2892 [INSPIRE].

[184] ATLAS collaboration, ATLAS Run 1 searches for direct pair production of third-generation squarks at the Large Hadron Collider, arXiv:1506.08616 [INSPIRE].

[185] CMS collaboration, Searches for third-generation squark production in fully hadronic final states in proton-proton collisions at $\sqrt{s}=8 \mathrm{TeV}$, JHEP 06 (2015) 116 [arXiv:1503.08037] [INSPIRE].

[186] ATLAS collaboration, Search for supersymmetry in events containing a same-flavour opposite-sign dilepton pair, jets and large missing transverse momentum in $\sqrt{s}=8 \mathrm{TeV} p p$ collisions with the ATLAS detector, Eur. Phys. J. C 75 (2015) 318 [arXiv:1503.03290] [INSPIRE].

[187] CMS collaboration, Searches for supersymmetry using the $M_{T 2}$ variable in hadronic events produced in pp collisions at $8 \mathrm{TeV}$, JHEP 05 (2015) 078 [arXiv: 1502.04358] [INSPIRE].

[188] ATLAS collaboration, Search for direct production of charginos, neutralinos and sleptons in final states with two leptons and missing transverse momentum in pp collisions at $\sqrt{s}=8 \mathrm{TeV}$ with the ATLAS detector, JHEP 05 (2014) 071 [arXiv:1403.5294] [INSPIRE].

[189] ATLAS collaboration, Searches for heavy long-lived sleptons and R-hadrons with the ATLAS detector in pp collisions at $\sqrt{s}=7$ TeV, Phys. Lett. B 720 (2013) 277 [arXiv:1211.1597] [INSPIRE].

[190] CMS collaboration, Searches for electroweak production of charginos, neutralinos and sleptons decaying to leptons and $W, Z$ and Higgs bosons in pp collisions at $8 \mathrm{TeV}$, Eur. Phys. J. C 74 (2014) 3036 [arXiv:1405.7570] [INSPIRE].

[191] CMS collaboration, Searches for electroweak neutralino and chargino production in channels with Higgs, $Z$ and $W$ bosons in pp collisions at $8 \mathrm{TeV}$, Phys. Rev. D 90 (2014) 092007 [arXiv: 1409.3168] [INSPIRE]. 